\title{
Contributions of Nonverbal Cues to the Accurate Judgment of Personality Traits
}

Simon M. Breil ${ }^{1}$, Sarah Osterholz ${ }^{2}$, Steffen Nestler ${ }^{1}, \&$ Mitja. D. Back ${ }^{1}$

\author{
${ }^{1}$ Department of Psychology, University of Münster, Germany \\ ${ }^{2}$ Department of Psychology, Johannes Gutenberg University Mainz, Germany
}

This is an unedited chapter that has been accepted for publication in the Oxford handbook of accurate personality judgment. The chapter will undergo copyediting, typesetting, and review of the resulting proof before it is published in its final form.

Breil, S. M., Osterholz, S., Nestler, S., \& Back, M. D. (2021). Contributions of nonverbal cues to the accurate judgment of personality traits. In T. D. Letzring \& J. S. Spain (Eds.), The Oxford handbook of accurate personality judgment (pp. 195-218). Oxford University Press. https://doi.org/10.1093/oxfordhb/9780190912529.013.13

\section{Author Note}

Correspondence concerning this manuscript should be addressed to Simon Breil (simon.breil@uni-muenster.de), University of Münster, Institute for Psychology, Psychological Assessment and Personality Psychology, Fliednerstraße 21, 48149 Münster, Germany) 


\begin{abstract}
In this chapter, we summarize research on nonverbal expressions of behavior (nonverbal cues) and how they contribute to the accuracy of personality judgments. First, we present a conceptual overview of relevant nonverbal cues in the domains of facial expressions, body language, paralanguage, and appearance as well as approaches to assess these cues on different levels of aggregation. We then summarize research on the validity of nonverbal cues (what kind of nonverbal cues are good indicators of personality?) as well as the utilization of nonverbal cues (what kind of nonverbal cues lead to personality impressions?), resulting in a catalogue of those cues that drive judgment accuracy for different traits. Finally, we discuss personal and situational characteristics that moderate the expression and utilization of nonverbal cues and give an outlook for future research.
\end{abstract}

Keywords: Judgment Accuracy, Personality Judgments, Nonverbal behavior, Behavioral Cues, Lens Model Analyses 
Whenever we interact with others, we make judgments about their personalities (e.g., this person is trustworthy, this person is friendly). These judgments are typically formed within seconds (e.g., Willis \& Todorov, 2006), tend to be quite stable across time (e.g., Kenny, Horner, Kashy, \& Chu, 1992), and are often surprisingly accurate (e.g., Ambady \& Skowronski, 2008; Funder, 2012). Most of the time, initial judgments are even formed before any (relevant) verbal information is exchanged. They are thus exclusively based on nonverbal cues (i.e., the way people look, move, or gesture) and they can have far-reaching consequences (e.g., Ambady, Bernieri, \& Richeson, 2000; Harris \& Garris, 2008). For example, in an employment interview, the applicant's upright posture, firm handshake, and appearance might lead to a positive evaluation (e.g., the applicant seems trustworthy and competent) and eventually to a job offer. In a get-to-know context, the broad smile of an interaction partner and the colorful clothing could lead to the conclusion that this person is friendly, thus resulting in a friendship or a romantic relationship.

In this chapter, we will present an overview of nonverbal cues and how they are related to the accuracy of personality judgments. That is, we will first summarize relevant nonverbal cue domains (i.e., facial expressions, body language, paralanguage, appearance) and discuss why they might be important for the judgment of personality and how they can be assessed. Afterwards, using the lens model as a conceptual framework, we will summarize their role in explaining the degree of accuracy in personality trait judgments. We will then present research on the validity (what kind of nonverbal cues are good indicators of 'true' personality?) and utilization (what kind of nonverbal cues are good indicators of personality impressions?) of nonverbal cues. Based on this, we will discuss potential moderators that might influence the relation between nonverbal cues and trait accuracy (i.e., good trait, good information, good judge, good target) and outline implications for future research. 


\section{Conceptualization of Nonverbal Cues}

There has been a long history of studying nonverbal (behavioral) cues, going as far back as Darwin (1897) who examined the expression of emotions through facial cues and gestures. Numerous studies in this spirit focused on nonverbal cues and their relation to judgments of emotions and personality (e.g., Ekman \& Friesen, 1969; Scherer, Scherer, Hall, \& Rosenthal, 1977; Taft, 1955; for overviews of nonverbal communication and behavioral research see: Burgoon, Guerrero, \& Floyd, 2010; Hall, Horgan, \& Murphy, 2018; Harrigan, Rosenthal, \& Scherer, 2005; Manusov, 2004). Nonverbal cues are typically divided into three domains of dynamic cues (i.e., cues that can easily be changed): face (i.e., facial expressions), body (i.e., body language; sometimes further divided into gestures and postures), and tone (i.e., paralanguage; Blanck, Rosenthal, Snodgrass, DePaulo, \& Zuckerman, 1981; Elfenbein \& Eisenkraft, 2010; Hall \& Andrzejewski, 2008; Hall, Schmidt Mast, \& West, 2016; Nowicki \& Duke, 1994). In addition to these dynamic cues, there also exists a long research tradition of investigating static appearance based cues (e.g., body shape, choice of clothing, head size; DeGroot \& Gooty, 2009; Gifford, Ng, \& Wilkinson, 1985; Naumann, Vazire, Rentfrow, \& Gosling, 2009; Scherer et al., 1977), which also play an important role in nonverbal expression. Cues based on environmental aspects such as rooms (Gosling, Ko, Mannarelli, \& Morris, 2002), music (Rentfrow \& Gosling, 2006), or verbal descriptions (e.g., Borkenau, Mosch, Tandler, \& Wolf, 2016; Küfner, Back, Nestler, \& Egloff, 2010), as well as social media cues (e.g., Back, Schmukle, \& Egloff, 2008; Back, Stopfer et al., 2010) will be discussed in Ch. 14 by Wall and Campbell in this handbook. Thus, for this chapter, we focus on facial expressions, body language, paralanguage, and appearance as four generally distinct domains of nonverbal behavior (please refer to Table 1 for an overview with examples). 
Table 1

Overview of nonverbal cue domains

\begin{tabular}{lll}
\hline Domain & Definition & Examples \\
\hline Facial expressions & Movements executed & Smiling \\
& with the facial muscles & Eye contact \\
& & Frowning \\
& & Winking \\
\hline Body language & Arrangement and & Trunk reclines \\
& gestures executed with & Energetic stance \\
& the body and its limbs & Body movement \\
& & Head shake \\
\hline Paralanguage & Nonverbal elements & Pitch \\
& and features of speech & Speech rate \\
& & Amplitude \\
& & Voice breaks \\
\hline Appearance & Static visual cues & Body shape \\
& Height \\
& & Stylish clothes \\
& & Make up \\
\hline
\end{tabular}

\section{Facial expressions}

Facial expressions refer to any kind of movements with the facial muscles. This most prominently includes expressions via the mouth and lips (e.g., smiling, yawning, showing teeth) as well as expressions via the eyes and eyebrows (e.g., eye contact, glazing, squinting, winking, scowling). Research on such facial expressions has predominantly focused on the recognition of emotions (e.g., Ekman \& Friesen, 1969, 1971; Ekman, Sorenson, \& Friesen, 1969; Izard, 1971), which are displayed through an interplay of different facial muscle movements. For example, the emotion of anger can be characterized by pulled-down eyebrows, pulled-up eyelids, and tightened lips (for an overview see: Ekman \& Rosenberg, 1997). While the kind of emotion signals are often assumed to be universal across cultures 
(Ekman, 1994, 2016; Izard, 1994), there is large variability between individuals of how strongly emotions are expressed (e.g., Hildebrandt, Olderbak, \& Wilhelm, 2015).

Individual differences in facial expressions, whether they are used for the recognition of emotions or not, can be used as cues for the judgment of underlying personality traits. One possible explanation lies in the relation between facial cues, affect, and traits. The State and Trait Accuracy Model (STAM; Hall, Gunnery, Letzring, Carney, \& Colvin, 2017), for example, posits that people first judge affective states (e.g., a person with pulled-down eyebrows might be perceived as angry, a person with a wide smile might be perceived as happy). These affective state impressions might then be used to form trait judgments (e.g., an angry person could be seen as disagreeable, a happy person could be seen as extraverted). This is based on the idea that momentary characteristics are often regarded as enduring attributes (i.e., the process of temporal extension; Knutson, 1996; Secord, 1958; Zebrowitz \& Montepare, 2008). This link between facial expressions, emotions, and personality judgments has been shown in a variety of studies (for an overview, see: Zebrowitz \& Montepare, 2008) and serves as a starting point for the understanding of accurate trait judgments based on facial expressions.

\section{Body language}

Body language involves any kind of arrangement or gestures performed with the body and its limbs (i.e., every movement or arrangement of a person except the facial muscles). This includes gestures performed with the arms and hands (e.g., folded arms, waving, itching, peace sign), movement with the legs (e.g., running, walking) or head (e.g., head shake, head pointed towards the ground), as well as arrangements concerning the whole body (e.g., upright posture, energetic stance, crouched position). Some of these movements and gestures have concrete meanings within specific cultural groups (often called emblems, e.g., in many cultures a head shake stands for 'no' and a thumbs up stands for 'I agree', cf. Hall et al., 2018). Research on body-related cues has often focused on the display, recognition, and 
relationship with dominance, power, and status (e.g., Carney, Hall, \& LeBeau, 2005; Hall, Coats, \& LeBeau, 2005; Henley, 1977). For example, upright postures, upright head tilts, and wide gestures are generally seen as signs of dominance.

Again, differences in the expression of such behaviors can be used as cues for the judgment of underlying traits. In addition to previously mentioned explanations (i.e., temporal extension, STAM) of how differences in expression can be related to (more or less accurate) personality judgments, body language cues especially can function via the accurate perception of underlying goals and motives. An individual who shows cues such as a huddled posture or head pointed towards the ground might, for example, be seen as someone with a (conflict) avoidance motive that wants to maintain a stable relationship with possible interaction partners. This person might then be (correctly) identified as being submissive or introverted (cf. Hall et al., 2005 for multiple explanations of how social pressures, goals, motives, emotions, and contextual variables impact nonverbal cues in the context of dominance).

\section{Paralanguage}

Paralanguage (i.e., paraverbal cues) describes nonverbal elements and general features of speech such as speech rate (number of words within a specific timeframe), voice breaks (number of pauses), fundamental frequency (mean voice pitch), pitch variability (variation of the voice pitch), amplitude (mean loudness/intensity of the voice), or amplitude variability (variation in the loudness of the voice). These vocal cues often parallel the spoken word, but can also provide additional (e.g., emotional state) or contradictory (e.g., sarcasm, deception) information (Hall et al., 2018). There is a large amount of research focusing on the perception of different paralanguage cues which shows that people generally rely heavily on voice and speech features when forming impressions about others (e.g., DeGroot \& Gooty, 2009; Kramer, 1963). One example is the relationship between cognitive ability and paralanguage: Perceptions made via acoustic cues (within a standardized content, e.g., reading the weather forecast) have been shown to relate to differences in individuals' cognitive ability measured 
via standardized intelligence tests (Borkenau \& Liebler, 1993, 1995; Borkenau, Mauer, Riemann, Spinath, \& Angleitner, 2004).

This can most likely be explained by the fact that ability measured in cognitive tests (e.g., rapid information processing, good working memory) is also important for the comprehension and fluent repetition of words and sentences (i.e., reading, see for example: Borkenau \& Liebler, 1995). These possible differences in speech rate, pauses, and emphasis might then be used by observers as cues for competence. Of course, paralinguistic cues can also carry information about emotions and affective states (e.g., speaking slowly and quietly might be a sign for a sad mood, cf. Bänziger, Hosoya, \& Scherer, 2015), as well as reveal individual motives of how one wants to appear to others that are based on stereotypes (e.g., Anna believes herself to be dominant, thus she general speaks in a loud voice when talking to others).

\section{Appearance}

Nonverbal cues relating to appearance include any static visual cues. These are physical characteristics that are usually less alterable such as height, weight, body shape, length of nose, width of chin, shape of ears, eye color, or hair color as well as more alterable features like choice of clothing, grooming, hairstyle, or makeup. All appearance-based cues have in common, however, that they are generally static within one situation and, in contrast to facial expressions and body language, cannot be easily changed within the specific judgment context. Theories and research about the relationship between static visual cues and personality dates back to physiognomical ideas of ancient Greek philosophers more than 2000 years ago (cf. Hassin \& Trope, 2000; Zebrowitz, 1997), who believed that there are 'mystic' links between a person's character and outer appearance. While these simplified ideas have been heavily criticized, newer research has focused on more specific relationships between appearance characteristics and personality, and there are hints that appearance can provide us 
with at least rudimentary guidelines when judging personality (i.e., a kernel of truth; e.g., Berry, 1990; Naumann et al., 2009; Zebrowitz, 2017; Zebrowitz \& Montepare, 2008).

Associations between personality and appearance can be traced back to a variety of mechanisms involving environmental and biological factors. For example, appearance and personality might be related due to common biological causes (e.g., testosterone influences facial hair growth as well as a more aggressive personality) as well as common environmental causes (e.g., the choice for a specific grooming style is specific for individuals that share the same values). Furthermore, physical differences could serve as some form of self-fulfilling prophecy (e.g., tall individuals are expected to behave more dominantly, they thus act accordingly) or they could reveal differences in past behaviors (e.g., individuals that laugh a lot might develop laugh lines and are thus judged to be happy or extraverts; see Zebrowitz \& Collins, 1997 for an overview of these four explanations).

\section{Assessment of Nonverbal Cues}

To empirically investigate the contribution of nonverbal cues to trait accuracy one needs three different data sources: This includes some measure of "real" personality provided by the targets (typically assessed via self-reported personality questionnaires and sometimes supplemented by informant-reported personality), judgments on the same personality traits by unacquainted perceivers (typically based on short interactions, videos, or photos), as well as a listing and quantification of nonverbal cues available to perceivers. At best, these cues should be sampled with respect to their natural range and covariation and rated by independent observers.

Generally, the assessment of such cues can happen at different levels of abstraction which include the macro level (global ratings specific to one behavioral domain; e.g., shows dominant behavior), meso level (circumscribed behavioral expressions; e.g., self-confident facial expressions) and micro level (specific behavioral acts; e.g., leaning forward). The specific cues that are to be rated are typically based on coding schemes developed for the 
assessment of (nonverbal) cues (for overviews see: Bakemann, 2000; Brauner, Boos, \& Kolbe, 2018; Heyman, Lorber, Eddy, \& West, 2014; Manusov, 2004). These schemes vary in their number of cues and comprehensiveness. The Münster Behavior Coding System (Grünberg, Mattern, Geukes, Küfner, \& Back, 2018), for example, differentiates between the mentioned macro, meso, and micro levels and classifies cues within the broader sections of dominance, warmth, expressiveness, arrogance, aggressiveness, nervousness, and intellectual competence, resulting in over 280 possible cues. When used for specific research questions the number of assessed cues is often considerably lower. An example are the classic studies by Borkenau and Liebler (1992a, 1992b, 1995) in which observers rated about 50 behavioral and appearance-based cues.

Cues further differ in how they can be assessed: there are cues that can be objectively determined (e.g., target wears glasses, height of target, body proportions), cues that can be counted (e.g., number of aggressive gestures, number of smiles), and cues that are rated on Likert type-scales (e.g., extent of leaning forward, extent of eye contact). Due to the sheer number of cues investigated, live rating is not an option in most cases. Thus, usually multiple observers need to watch videos or evaluate photos of targets to assess all desired nonverbal cues.

\section{Role of Nonverbal Cues for Accuracy}

How are nonverbal cues related to trait accuracy when judging personality traits? Based on the assumptions of the lens model (Brunswik, 1952, 1956 see Ch. 4 by Osterholz, Breil, Nestler, \& Back in this handbook for a detailed explanation; see Figure 1 for an example), perceivers use available cues in the environment to form judgments of personality traits that are not directly observable. This framework enables an analytic and differentiated analysis of involved processes. 


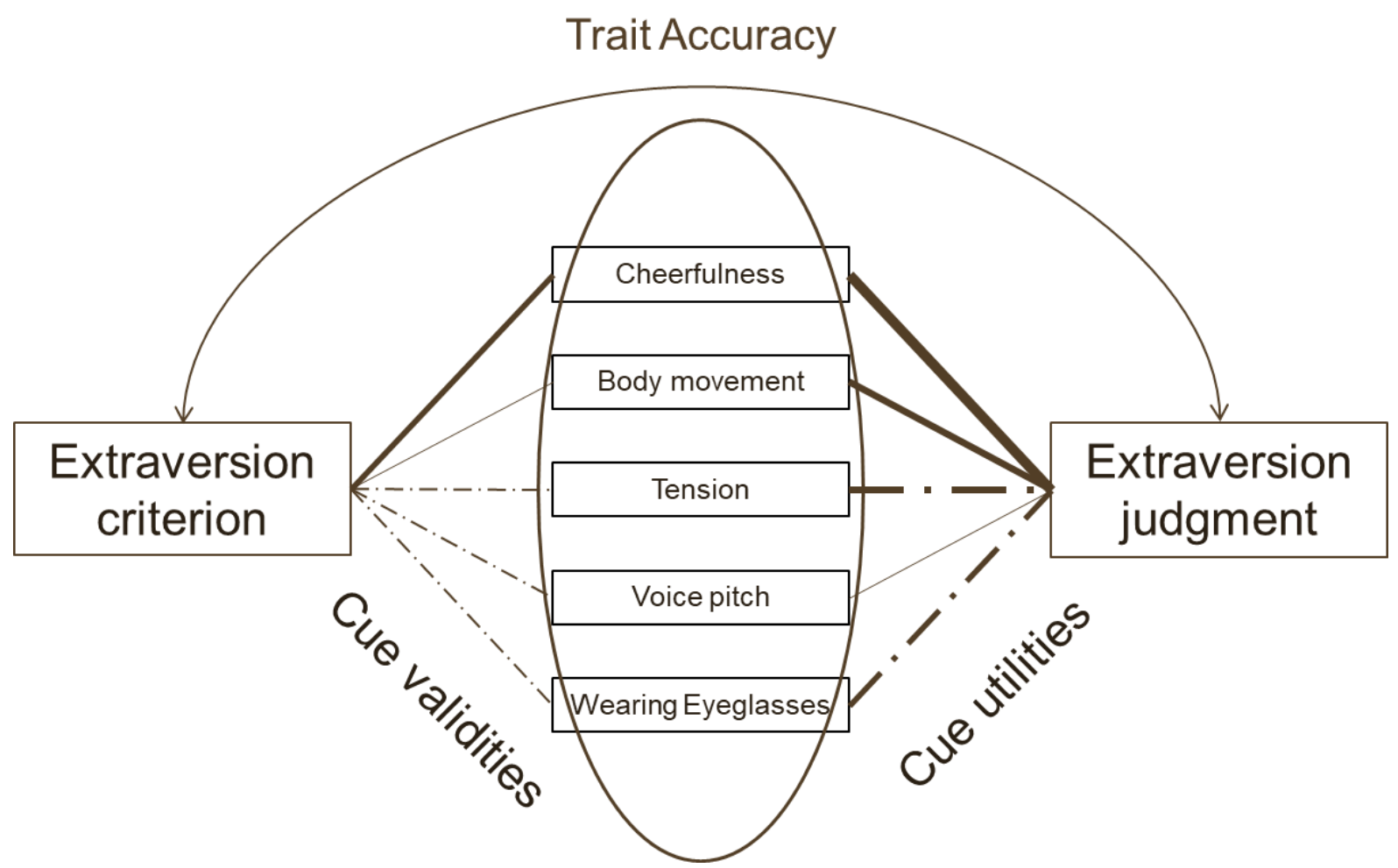

Figure 1. Exemplary lens model for the judgment of personality traits. The thickness of the lines indicates the strength of the relation. Solid lines indicate positive associations, dotted lines indicate negative associations.

For example, perceivers might observe the cheerfulness, the amount of body movement, and the lack of tension of a target and use this information to infer his/her (possibly high) extraversion. The extent to which an observer uses a specific cue for judgments is called cue utilization (see right side in Figure 1). For each cue, it describes the relationship between individual differences in target cue values and individual differences in how targets are judged. Cue utilizations are influenced by (intuitive) knowledge and individual experiences, as well as the utilization of stereotypes (e.g., Jussim, Cain, Crawford, Harber, \& Cohen, 2009) and exemplar information (e.g., Smith \& Zárate, 1992).

Cue validity on the other side, describes, for each cue, the relationship between individual differences in targets (nonverbal) cue values and individual differences in targets actual personality trait values (typically assessed via self-report and/or informant-report questionnaires; see left side in Figure 1). Individuals who wear glasses, for example, might be 
(on average) less extraverted, while individuals who smile a lot (i.e., have a cheerful expression) might be more extraverted. Multiple (not mutually exclusive) explanations on why differences in the expression of nonverbal cues could be related to differences in actual personality traits have been discussed above and are displayed in Table 2. Nonverbal cues especially might be very suited for the judgment of personality as they are more difficult to suppress relative to verbal behavior and are thus more revealing of internal states (DePaulo, 1992). 
Table 2

Explanations on why nonverbal cues are related to "real" personality

\begin{tabular}{lll}
\hline Reason & Explanation & Potential Examples \\
\hline & Individuals who are high in specific traits are more & Extraverts are more likely to be happy, thus they smile more often.
\end{tabular}

Temporal extension likely to show specific emotions and affective states.

of emotion and affect These emotions and affective states are expressed People high in neuroticism are more likely to be afraid, thus their through nonverbal cues.

body is shaking more often.

Individuals who are high in specific traits are more Introverts are more likely to have a conflict avoidance motive, thus likely to have specific motives and goals when

Motives/Goals interacting with others. These motives, goals, and interests are expressed through nonverbal cues. Individuals high in openness are more likely to have goals related to

Individuals who are high in specific traits are more likely to show specific nonverbal cues based on genetic and biological links.

\section{Common biological}

cause the creative expression of oneself and are thus more likely to wear extravagant clothes.

they show submissive (gestures, facial expressions) behavior.

Aggressive persons are more likely to have strong beard growth (due

to testosterone which influences facial hair growth and aggressiveness).

Intelligent individuals are more likely to be generally good looking

(e.g., possible due to "good genes"). 


\begin{tabular}{lll}
\hline $\begin{array}{l}\text { Common } \\
\text { environmental cause }\end{array}$ & $\begin{array}{l}\text { Individuals who are high in specific traits are more } \\
\text { likely to show specific nonverbal cues based on } \\
\text { correlated learning. }\end{array}$ & $\begin{array}{l}\text { Individuals low in openness have generally learned conservative } \\
\text { values and, thus, wear more formal (conservative) clothes. }\end{array}$ \\
\hline $\begin{array}{l}\text { Self-fulfilling } \\
\text { prophecy }\end{array}$ & $\begin{array}{l}\text { Individuals who are high in specific traits are more } \\
\text { likely to show specific nonverbal cues based on self- } \\
\text { fulfilling prophecy which causes them to behave in a } \\
\text { manner consistent with expectations. }\end{array}$ & $\begin{array}{l}\text { Taller individuals are more likely to be dominant because they act } \\
\text { act and thus, have a slower speech rate. }\end{array}$ \\
& $\begin{array}{l}\text { Individuals who are high in specific traits are more } \\
\text { likely to show specific nonverbal cues based on past } \\
\text { behavior that led to the development of these cues. }\end{array}$ & $\begin{array}{l}\text { Individuals low in conscientiousness are more likely to be overweight } \\
\text { because they behaved less conscientiously in the past. }\end{array}$ \\
\hline
\end{tabular}

Note. For more information on these different explanations please refer to Hall et al., 2005; Hall et al., 2017; Zebrowitz \& Collins, 1997; Zebrowitz

\& Montepare, 2008. Please note that the provided examples just serve as a representation of different mechanisms and not all links have been

empirically investigated. Furthermore, the mechanisms are not mutually exclusive (e.g., a common environmental cause might influence motives and goals). 
In summary, accuracy through nonverbal cues can be achieved when there are valid cues available in the environment, that are observable, and used in line with their degree of validity. If there are no valid cues in the environment for a given trait, this means that this trait is impossible to judge accurately within this specific context. If there are valid cues, but they are not used accordingly by perceivers, this would indicate that perceivers have missing knowledge. The utilization of cues in the absence of validity could suggest some sort of bias or false stereotype.

\section{Empirical Results for Validity and Utilization of Nonverbal Cues}

\section{Study selection and procedure}

In this part, we will summarize empirical results regarding the validity and utilization of nonverbal cues for personality traits. We decided to include results of studies investigating the relationship between nonverbal cues on the one side and personality and/or personality judgments on the other side. We hereby only included studies with 1) real targets (age $\geq 16$ ), with 2) a natural range and covariation of cues (i.e., no experimental manipulation), with 3) non-evaluative traits (e.g., no liking or popularity), and with 4) cues that classify to one of the four categories (i.e., facial expression, body language, paralanguage, appearance; e.g., no environmental cues, no highly aggregated cues).

We identified relevant studies through multiple criteria: In a first step we scanned known studies (and cross-references within these studies) that focused on the lens model, on cue-based judgments, and on behavioral prediction. In total, 32 studies met our criteria. In a second step, we used EBSCO, including the databases PsycARTICLES, PsycINFO, PSYINDEX, PsycBOOKS, and MEDLINE, for an online literature search. The search was restricted to peer-reviewed journals. Keywords were lens model [AND] nonverbal (all fields, 17 results), nonverbal cues [AND] personality (key words only, 30 results), personality judgment (key words only, 271 results), personality perception [AND] cues (all fields, 57 results), and big five [AND] nonverbal (all fields, 58 results). Here we found 10 additional 
studies. In a last step, we scanned the first dozen pages of Google Scholar using the same criteria and identified 22 additional articles. In total, 64 articles were included (see osf.io/9p64g, Table S1) for an overview of included studies with relevant characteristics. Please note that some articles included multiple studies, while other studies were covered by multiple articles, resulting in 65 independent studies. Of these 65 studies, 33 studies included both cue validities as well as cue utilizations. Fifteen studies included only validities, while 17 studies exclusively focused on utilizations.

On the OSF page of this chapter (osf.io/9p64g), we have uploaded the full table with all studies and all individual correlations. Please note, however, that this list does not aim to be fully comprehensive and should be regarded as a preliminary documentation of the existing work. On the same page, we have included a sheet in which additional relevant studies we might have missed can be added, and we invite all readers to contribute to a more exhaustive documentation on personality (judgments) and nonverbal cues.

For the presentation of results in this chapter, we have focused on the relationship (correlation) between the Big Five (supplemented by intelligence) and 39 cues (6 to 14 per domain), which were selected based on their number of occurrences across studies. We hereby summarized traits and cues to broader categories (e.g., dominance and shyness were allocated to extraversion; warmth and arrogance were allocated to agreeableness; smiles and happy expression were allocated to cheerful facial expression; stylish hair and fashionable dress were allocated to stylishness). This allocation process was done by the authors of this study and based on theoretical (e.g., childlike, feminine, and soft faces are all related to babyfaceness) and practical (e.g., to have multiple studies for every trait / cue combination) considerations (also see Hirschmüller, Schmukle, Krause, Back, \& Egloff, 2018 for a similar allocation of cues). For an overview concerning the current allocation of traits and cues, please refer to the online supplement (osf.io/9p64g, Table S2). ${ }^{1}$ 
As some studies did not provide zero-order correlations, we used the provided partial correlations for those studies (e.g., correlations controlled for sex and age). When there were multiple correlations for a specific trait and cue combination within a sample (e.g., multiple traits within a sample allocated to extraversion, multiple cues within a study allocated to stylishness, separate results for men and women, self- and informant-reported traits), we aggregated these correlations within studies. In a next step, we meta-analyzed the resulting correlations across studies, using the R package meta (Schwarzer, 2019; R version 3.4.3, see osf.io/9p64g for data and code). Reported estimations are based on a random effects model with inverse variance weighting and DerSimonian-Laird estimation for the between-study variance. Please refer to Tables 3 to 8 for results. 
Table 3

Cue Validity and Cue Utilization Meta-Analyses: Neuroticism

\begin{tabular}{|c|c|c|c|c|c|c|}
\hline \multicolumn{3}{|c|}{ Cue Validity } & \multirow{2}{*}{$\frac{\text { Neuroticism }}{\text { Cues }}$} & \multicolumn{3}{|c|}{ Cue Utilization } \\
\hline$k(n)$ & $95 \% \mathrm{CI}$ & $r$ & & $r$ & $95 \% \mathrm{CI}$ & $k(\mathrm{n})$ \\
\hline & & & Facial expression & & & \\
\hline $9(862)$ & {$[-.18,-.03]$} & -.11 & Cheerful facial expression & -.24 & {$[-.46, .02]$} & $9(878)$ \\
\hline $4(414)$ & {$[-.16, .04]$} & -.06 & Dominant facial expression & -.58 & {$[-.74,-.36]$} & $2(200)$ \\
\hline $1(62)$ & & -.15 & General expressiveness & $\mathrm{n} / \mathrm{a}$ & $\mathrm{n} / \mathrm{a}$ & $\mathrm{n} / \mathrm{a}$ \\
\hline $3(299)$ & {$[-.16, .11]$} & -.03 & Unconcerned (vs. serious) & .10 & {$[-.42, .56]$} & $3(299)$ \\
\hline $7(524)$ & {$[-.19, .07]$} & -.07 & Eye contact & -.14 & {$[-.24,-.04]$} & $4(388)$ \\
\hline $2(116)$ & {$[-.55, .85]$} & .31 & Eyebrow movements & -.01 & {$[-.19, .18]$} & $2(116)$ \\
\hline & & & Body language & & & \\
\hline $5(498)$ & {$[-.20, .08]$} & -.06 & Body movement & .09 & {$[-.18, .35]$} & $6(466)$ \\
\hline $3(365)$ & {$[-.24, .12]$} & -.06 & Forward lean / proximity & $\mathrm{n} / \mathrm{a}$ & $\mathrm{n} / \mathrm{a}$ & $\mathrm{n} / \mathrm{a}$ \\
\hline $5(420)$ & {$[-.12, .07]$} & -.03 & Gestures & -.12 & {$[-.25, .02]$} & $2(200)$ \\
\hline $3(189)$ & {$[-.27, .08]$} & -.10 & Head movements & .13 & {$[-.21, .44]$} & $2(140)$ \\
\hline $4(336)$ & {$[-.16, .14]$} & -.01 & Self-assured / open vs. slouching & -.40 & {$[-.64,-.09]$} & $3(224)$ \\
\hline $3(313)$ & {$[-.06, .17]$} & .06 & Closed arms & .13 & {$[-.01, .27]$} & $3(313)$ \\
\hline $3(208)$ & {$[-.14, .13]$} & -.01 & Self-touch & .17 & {$[-.29, .57]$} & $2(164)$ \\
\hline $4(325)$ & {$[.02, .23]$} & .13 & Tension / nervousness (vs. relaxed) & .29 & {$[.00, .53]$} & $4(325)$ \\
\hline $2(200)$ & {$[-.09, .19]$} & .05 & Stride length & -.13 & {$[-.28, .02]$} & $2(200)$ \\
\hline & & & Paralanguage & & & \\
\hline $2(200)$ & {$[-.09, .19]$} & .05 & Ease of understanding & -.33 & {$[-.51,-.14]$} & $2(200)$ \\
\hline $2(123)$ & {$[-.43,-.02]$} & -.24 & Expressive / varying voice & -.47 & {$[-.70,-.14]$} & $3(180)$ \\
\hline $7(698)$ & {$[-.20,-.01]$} & -.10 & Fluent speaking (vs. nervous) & -.32 & {$[-.40,-.24]$} & $5(510)$ \\
\hline $3(299)$ & {$[-.23, .00]$} & -.12 & Pleasantness of voice & -.50 & {$[-.60,-.39]$} & $4(323)$ \\
\hline $3(299)$ & {$[-.19, .04]$} & -.07 & Powerful / confident voice (vs. soft) & -.28 & {$[-.41,-.13]$} & $4(323)$ \\
\hline $2(248)$ & {$[-.29, .02]$} & -.14 & Loudness & -.17 & {$[-.45, .13]$} & $3(180)$ \\
\hline $3(299)$ & {$[-.25, .23]$} & -.01 & Pitch & .08 & {$[-.07, .22]$} & $6(822)$ \\
\hline $1(54)$ & & .20 & Speech rate & -.20 & {$[-.46, .10]$} & 2(499) \\
\hline $5(446)$ & {$[-.32, .07]$} & -.13 & Speech vs. non-speech & -.11 & {$[-.19,-.03]$} & $3(623)$ \\
\hline $\mathrm{n} / \mathrm{a}$ & $\mathrm{n} / \mathrm{a}$ & $\mathrm{n} / \mathrm{a}$ & Interruptions & $\mathrm{n} / \mathrm{a}$ & $\mathrm{n} / \mathrm{a}$ & $\mathrm{n} / \mathrm{a}$ \\
\hline & & & Appearance & & & \\
\hline $9(865)$ & {$[-.22,-.06]$} & -.14 & Attractiveness & -.33 & {$[-.45,-.19]$} & $10(843)$ \\
\hline $6(476)$ & {$[-.12, .10]$} & -.01 & Babyfaceness vs. maturity & -.01 & {$[-.26, .24]$} & $4(276)$ \\
\hline $3(189)$ & {$[-.11, .18]$} & .04 & Distinctiveness & .00 & {$[-.14, .15]$} & $3(189)$ \\
\hline $9(764)$ & {$[-.17,-.02]$} & -.10 & Neatness & -.15 & {$[-.24,-.05]$} & $10(757)$ \\
\hline $8(752)$ & {$[-.11, .03]$} & -.04 & Stylishness & -.15 & {$[-.28,-.02]$} & $8(690)$ \\
\hline $3(212)$ & {$[-.24, .24]$} & .00 & Formality & -.08 & {$[-.21, .06]$} & $3(212)$ \\
\hline $3(149)$ & {$[-.22, .30]$} & .04 & Eyeglasses & .24 & {$[.01, .45]$} & $2(76)$ \\
\hline $3(176)$ & {$[-.10, .20]$} & .06 & Volume of Mouth / full lips & -.05 & {$[-.39, .31]$} & $3(176)$ \\
\hline $2(200)$ & {$[-.09, .19]$} & .05 & Hair length (long) & .11 & {$[-.03, .24]$} & $2(200)$ \\
\hline $3(176)$ & {$[.10, .38]$} & .25 & Dark hair color & .01 & {$[-.14, .16]$} & $3(176)$ \\
\hline $2(199)$ & {$[-.24, .04]$} & -.10 & Height & -.18 & {$[-.32,-.05]$} & $2(199)$ \\
\hline $3(273)$ & {$[-.24, .00]$} & -.12 & Muscular & -.33 & {$[-.49,-.14]$} & $2(200)$ \\
\hline $3(299)$ & {$[-.05, .18]$} & .07 & Weight & -.04 & {$[-.29, .21]$} & $3(299)$ \\
\hline $2(200)$ & {$[.05, .32]$} & .19 & Dark clothes & .08 & {$[-.07, .21]$} & $2(200)$ \\
\hline
\end{tabular}

Note. $k=$ number of included samples, $n=$ overall sample size. The effect size and confidence interval (CI) estimations are based on a random effects model (empty CI cells included only one study). Cues in bold showed at least small ( $\mathrm{r} \geq .10)$ effects for both utilization and validity (same direction; across at least two studies). 
Table 4

Cue Validity and Cue Utilization Meta-Analyses: Extraversion

\begin{tabular}{|c|c|c|c|c|c|c|}
\hline \multicolumn{3}{|c|}{ Cue Validity } & Extraversion & \multicolumn{3}{|c|}{ Cue Utilization } \\
\hline$k(n)$ & $95 \% \mathrm{CI}$ & $r$ & Cues & $r$ & $95 \% \mathrm{CI}$ & $k(\mathrm{n})$ \\
\hline & & & Facial expression & & & \\
\hline 17(1789) & {$[.09, .21]$} & .15 & Cheerful facial expression & .54 & {$[.32, .70]$} & $17(1565)$ \\
\hline 6(669) & {$[-.08, .27]$} & .10 & Dominant facial expression & .52 & {$[.38, .64]$} & 3(382) \\
\hline $3(311)$ & {$[.03, .25]$} & .14 & General expressiveness & .40 & {$[.11, .63]$} & $4(371)$ \\
\hline $3(382)$ & {$[-.35, .26]$} & -.05 & Unconcerned (vs. serious) & -.29 & {$[-.75, .37]$} & $3(382)$ \\
\hline $12(1222)$ & {$[-.02, .09]$} & .03 & Eye contact & .21 & {$[.11, .31]$} & $11(1202)$ \\
\hline 4(394) & {$[-.17, .10]$} & -.04 & Eyebrow movements & .07 & {$[-.24, .37]$} & 4(394) \\
\hline & & & Body language & & & \\
\hline $8(733)$ & {$[-.02, .20]$} & .09 & Body movement & .33 & {$[.16, .48]$} & $10(837)$ \\
\hline $7(893)$ & {$[.04, .24]$} & .14 & Forward lean / proximity & .10 & {$[.03, .18]$} & $5(670)$ \\
\hline $12(1226)$ & {$[.11, .24]$} & .18 & Gestures & .35 & {$[.22, .46]$} & $11(1048)$ \\
\hline $8(808)$ & {$[-.14, .13]$} & .00 & Head movements & .26 & {$[.17, .35]$} & $8(802)$ \\
\hline $5(520)$ & {$[-.01, .31]$} & .15 & Self-assured / open vs. slouching & .25 & {$[-.30, .67]$} & $4(395)$ \\
\hline $6(623)$ & {$[-.18, .05]$} & -.06 & Closed arms & -.12 & {$[-.23,-.01]$} & $6(623)$ \\
\hline $7(697)$ & {$[-.03, .12]$} & .04 & Self-touch & .07 & {$[-.08, .22]$} & $7(711)$ \\
\hline $7(713)$ & {$[-.24,-.08]$} & -.16 & Tension / nervousness (vs. relaxed) & -.42 & {$[-.58,-.23]$} & $8(773)$ \\
\hline $3(382)$ & {$[-.07, .13]$} & .03 & Stride length & .05 & {$[-.05, .15]$} & $4(425)$ \\
\hline & & & $\underline{\text { Paralanguage }}$ & & & \\
\hline $4(449)$ & {$[-.04, .22]$} & .09 & Ease of understanding & .30 & {$[.11, .47]$} & $4(449)$ \\
\hline $7(849)$ & {$[.08, .33]$} & .21 & Expressive / varying voice & .36 & {$[.20, .50]$} & $7(514)$ \\
\hline $5(564)$ & {$[-.03, .22]$} & .10 & Fluent speaking (vs. nervous) & .22 & {$[.14, .31]$} & $5(472)$ \\
\hline $4(455)$ & {$[.08, .26]$} & .17 & Pleasantness of voice & .30 & {$[.16, .42]$} & $4(406)$ \\
\hline $6(894)$ & {$[.13, .30]$} & .22 & Powerful / confident voice (vs. soft) & .36 & {$[.25, .46]$} & $5(473)$ \\
\hline $3(387)$ & {$[-.03, .43]$} & .21 & Loudness & .35 & {$[.09, .57]$} & $6(581)$ \\
\hline $5(821)$ & {$[-.15, .08]$} & -.04 & Pitch & .05 & {$[-.08, .17]$} & $7(972)$ \\
\hline $3(493)$ & {$[-.25, .52]$} & .16 & Speech rate & .21 & {$[-.10, .49]$} & $4(609)$ \\
\hline $9(932)$ & {$[.15, .32]$} & .23 & Speech vs. non-speech & .31 & {$[.15, .45]$} & $7(1191)$ \\
\hline $2(197)$ & {$[-.01, .27]$} & .13 & Interruptions & .05 & {$[-.09, .19]$} & $2(197)$ \\
\hline & & & Appearance & & & \\
\hline 13(1603) & {$[.14, .27]$} & .20 & Attractiveness & .47 & {$[.37, .56]$} & $15(1362)$ \\
\hline $8(731)$ & {$[-.04, .21]$} & .08 & Babyfaceness vs. maturity & .06 & {$[-.07, .19]$} & $6(627)$ \\
\hline 3(189) & {$[-.29, .05]$} & -.12 & Distinctiveness & -.09 & {$[-.23, .06]$} & 3(189) \\
\hline 11(1064) & {$[.15, .27]$} & .21 & Neatness & .25 & {$[.18, .32]$} & $11(1009)$ \\
\hline $10(964)$ & {$[.14, .31]$} & .22 & Stylishness & .32 & {$[.21, .41]$} & $9(829)$ \\
\hline $4(367)$ & {$[-.09, .11]$} & .01 & Formality & -.08 & {$[-.22, .05]$} & $4(463)$ \\
\hline $3(149)$ & {$[-.32, .07]$} & -.13 & Eyeglasses & -.35 & {$[-.54,-.13]$} & $2(76)$ \\
\hline $4(358)$ & {$[.01, .22]$} & .12 & Volume of Mouth / full lips & .16 & {$[.05, .27]$} & $4(358)$ \\
\hline $2(282)$ & {$[-.14, .33]$} & .10 & Hair length (long) & .10 & {$[-.02, .22]$} & $2(282)$ \\
\hline $4(358)$ & {$[-.18, .06]$} & -.06 & Dark hair color & -.09 & {$[-.19, .01]$} & $4(358)$ \\
\hline $3(382)$ & {$[-.19, .19]$} & .00 & Height & .02 & {$[-.08, .12]$} & $3(382)$ \\
\hline $4(455)$ & {$[-.06, .12]$} & .03 & Muscular & .19 & {$[.02, .35]$} & $3(382)$ \\
\hline $4(455)$ & {$[-.01, .18]$} & .08 & Weight & .07 & {$[-.03, .17]$} & $3(382)$ \\
\hline $4(455)$ & {$[-.28, .04]$} & -.12 & Dark clothes & -.11 & {$[-.27, .06]$} & $3(382)$ \\
\hline
\end{tabular}

Note. $k=$ number of included samples, $n=$ overall sample size. The effect size and confidence interval (CI) estimations are based on a random effects model (empty CI cells included only one study). Cues in bold showed at least small ( $\mathrm{r} \geq .10)$ effects for both utilization and validity (same direction; across at least two studies). 
Table 5

Cue Validity and Cue Utilization Meta-Analyses: Openness

\begin{tabular}{|c|c|c|c|c|c|c|}
\hline \multicolumn{3}{|c|}{ Cue Validity } & \multirow{2}{*}{$\begin{array}{c}\text { Openness } \\
\text { Cues }\end{array}$} & \multicolumn{3}{|c|}{ Cue Utilization } \\
\hline$k(n)$ & $95 \% \mathrm{CI}$ & $r$ & & $r$ & $95 \% \mathrm{CI}$ & $k(\mathrm{n})$ \\
\hline & & & Facial expression & & & \\
\hline $6(711)$ & {$[-.06, .09]$} & .02 & Cheerful facial expression & .38 & {$[.29, .46]$} & $9(1026)$ \\
\hline $5(596)$ & {$[-.04, .12]$} & .04 & Dominant facial expression & .36 & {$[.27, .45]$} & $3(382)$ \\
\hline $1(182)$ & & -.01 & General expressiveness & .22 & & $1(182)$ \\
\hline $3(382)$ & {$[-.09, .11]$} & .01 & Unconcerned (vs. serious) & -.28 & {$[-.57, .08]$} & $3(382)$ \\
\hline $5(645)$ & {$[-.20, .06]$} & -.07 & Eye contact & .13 & {$[.00, .26]$} & $6(832)$ \\
\hline $2(116)$ & {$[-.26, .10]$} & -.08 & Eyebrow movements & .09 & {$[-.05, .22]$} & $3(326)$ \\
\hline & & & Body language & & & \\
\hline $4(394)$ & {$[-.13, .07]$} & -.03 & $\overline{\text { Body movement }}$ & .02 & {$[-.08, .11]$} & $5(434)$ \\
\hline $1(182)$ & & .02 & Forward lean / proximity & .15 & {$[.05, .24]$} & $2(392)$ \\
\hline $3(394)$ & {$[-.07, .13]$} & .03 & Gestures & .08 & {$[-.01, .17]$} & $3(492)$ \\
\hline $3(382)$ & {$[-.12, .09]$} & -.01 & Head movements & .07 & {$[-.06, .19]$} & $5(632)$ \\
\hline $3(237)$ & {$[-.03, .23]$} & .10 & Self-assured / open vs. slouching & .25 & {$[-.07, .52]$} & $3(335)$ \\
\hline $4(495)$ & {$[-.13, .06]$} & -.03 & Closed arms & -.10 & {$[-.18,-.01]$} & $4(495)$ \\
\hline $2(282)$ & {$[-.16, .21]$} & .02 & Self-touch & .02 & {$[-.10, .13]$} & $2(282)$ \\
\hline $5(507)$ & {$[-.13, .05]$} & -.04 & Tension / nervousness (vs. relaxed) & -.16 & {$[-.26, .05]$} & $5(507)$ \\
\hline $3(382)$ & {$[-.13, .13]$} & .00 & Stride length & -.01 & {$[-.27, .24]$} & $3(382)$ \\
\hline & & & $\underline{\text { Paralanguage }}$ & & & \\
\hline $3(382)$ & {$[-.09, .14]$} & .02 & Ease of understanding & .31 & {$[.11, .49]$} & $3(382)$ \\
\hline $\mathrm{n} / \mathrm{a}$ & $\mathrm{n} / \mathrm{a}$ & $\mathrm{n} / \mathrm{a}$ & Expressive / varying voice & $\mathrm{n} / \mathrm{a}$ & $\mathrm{n} / \mathrm{a}$ & $\mathrm{n} / \mathrm{a}$ \\
\hline $4(520)$ & {$[-.13, .08]$} & -.02 & Fluent speaking (vs. nervous) & .21 & {$[.12, .29]$} & $4(533)$ \\
\hline $3(382)$ & {$[.00, .20]$} & .10 & Pleasantness of voice & .44 & {$[.28, .58]$} & $3(382)$ \\
\hline $3(382)$ & {$[-.24, .10]$} & -.07 & Powerful / confident voice (vs. soft) & .10 & {$[-.03, .23]$} & $3(382)$ \\
\hline $1(182)$ & & -.18 & Loudness & -.23 & & $1(182)$ \\
\hline $3(382)$ & {$[-.18, .07]$} & -.06 & Pitch & .08 & {$[-.10, .25]$} & $4(824)$ \\
\hline $\mathrm{n} / \mathrm{a}$ & $\mathrm{n} / \mathrm{a}$ & $\mathrm{n} / \mathrm{a}$ & Speech rate & .05 & & $1(442)$ \\
\hline $3(432)$ & {$[-.32,-.02]$} & -.17 & Speech vs. non-speech & .06 & {$[-.01, .14]$} & $3(775)$ \\
\hline $1(182)$ & & -.04 & Interruptions & -.16 & & $1(182)$ \\
\hline & & & Appearance & & & \\
\hline $8(788)$ & {$[-.14, .09]$} & -.03 & Attractiveness & .56 & {$[.21, .78]$} & $9(828)$ \\
\hline $6(571)$ & {$[-.02, .14]$} & .06 & Babyfaceness vs. maturity & .17 & {$[.01, .31]$} & $5(458)$ \\
\hline $3(189)$ & {$[-.30, .25]$} & -.03 & Distinctiveness & .05 & {$[-.34, .42]$} & $3(189)$ \\
\hline $8(687)$ & {$[-.22, .09]$} & -.07 & Neatness & .32 & {$[.12, .49]$} & $10(840)$ \\
\hline $7(675)$ & {$[-.13, .14]$} & .00 & Stylishness & .18 & {$[.06, .29]$} & $7(675)$ \\
\hline $4(394)$ & {$[-.32, .08]$} & -.12 & Formality & .08 & {$[-.20, .35]$} & $4(394)$ \\
\hline $2(76)$ & {$[-.18, .28]$} & .05 & Eyeglasses & .00 & {$[-.23, .23]$} & $2(76)$ \\
\hline $4(358)$ & {$[.00, .33]$} & .17 & Volume of Mouth / full lips & .21 & {$[.01, .39]$} & $4(358)$ \\
\hline $2(282)$ & {$[-.02, .22]$} & .10 & Hair length (long) & .16 & {$[.05, .28]$} & $2(282)$ \\
\hline $5(458)$ & {$[-.05, .14]$} & .04 & Dark hair color & .09 & {$[-.01, .18]$} & $5(458)$ \\
\hline $3(382)$ & {$[-.09, .11]$} & .01 & Height & .12 & {$[.02, .22]$} & $3(382)$ \\
\hline $3(382)$ & {$[-.15, .05]$} & -.05 & Muscular & .02 & {$[-.29, .32]$} & $3(382)$ \\
\hline $3(382)$ & {$[-.18, .02]$} & -.09 & Weight & -.22 & {$[-.38,-.04]$} & $3(382)$ \\
\hline $3(382)$ & {$[-.01, .19]$} & .09 & Dark clothes & .05 & {$[-.05, .15]$} & $3(382)$ \\
\hline
\end{tabular}

Note. $k=$ number of included samples, $n=$ overall sample size. The effect size and confidence interval (CI) estimations are based on a random effects model (empty CI cells included only one study). Cues in bold showed at least small ( $\mathrm{r} \geq .10)$ effects for both utilization and validity (same direction; across at least two studies). 
Table 6

Cue Validity and Cue Utilization Meta-Analyses: Agreeableness

\begin{tabular}{|c|c|c|c|c|c|c|}
\hline \multicolumn{3}{|c|}{ Cue Validity } & \multirow{2}{*}{$\frac{\text { Agreeableness }}{\text { Cues }}$} & \multicolumn{3}{|c|}{ Cue Utilization } \\
\hline$k(n)$ & $95 \% \mathrm{CI}$ & $r$ & & $r$ & $95 \% \mathrm{CI}$ & $k(\mathrm{n})$ \\
\hline & & & Facial expression & & & \\
\hline $13(1225)$ & {$[.02, .19]$} & .10 & Cheerful facial expression & .53 & {$[.39, .64]$} & $17(1561)$ \\
\hline $4(385)$ & {$[-.27,-.06]$} & -.17 & Dominant facial expression & .22 & {$[.07, .37]$} & $2(200)$ \\
\hline $1(62)$ & & .09 & General expressiveness & $\mathrm{n} / \mathrm{a}$ & $\mathrm{n} / \mathrm{a}$ & $\mathrm{n} / \mathrm{a}$ \\
\hline $2(200)$ & {$[-.19, .09]$} & -.05 & Unconcerned (vs. serious) & -.26 & {$[-.39,-.12]$} & $2(200)$ \\
\hline $7(577)$ & {$[-.08, .08]$} & .00 & Eye contact & .24 & {$[.10, .36]$} & $8(814)$ \\
\hline $3(136)$ & {$[-.08, .26]$} & .09 & Eyebrow movements & -.04 & {$[-.14, .07]$} & $4(346)$ \\
\hline & & & Body language & & & \\
\hline $4(272)$ & {$[-.23, .07]$} & -.08 & Body movement & -.13 & {$[-.32, .06]$} & $7(394)$ \\
\hline $\mathrm{n} / \mathrm{a}$ & $\mathrm{n} / \mathrm{a}$ & $\mathrm{n} / \mathrm{a}$ & Forward lean / proximity & -.05 & {$[-.31, .22]$} & $3(330)$ \\
\hline $4(334)$ & {$[-.27, .17]$} & -.05 & Gestures & -.03 & {$[-.28, .22]$} & $4(430)$ \\
\hline $5(323)$ & {$[-.16, .28]$} & .06 & Head movements & .18 & {$[.00, .35]$} & $7(552)$ \\
\hline $4(310)$ & {$[-.20, .33]$} & .07 & Self-assured / open vs. slouching & .14 & {$[-.34, .56]$} & $4(395)$ \\
\hline $3(273)$ & {$[-.05, .19]$} & .07 & Closed arms & -.14 & {$[-.35, .09]$} & $3(273)$ \\
\hline $2(162)$ & {$[-.22, .19]$} & -.02 & Self-touch & -.10 & {$[-.37, .19]$} & $2(160)$ \\
\hline $4(325)$ & {$[-.26, .23]$} & -.02 & Tension / nervousness (vs. relaxed) & -.30 & {$[-.50,-.06]$} & $4(325)$ \\
\hline $2(200)$ & {$[-.41,-.01]$} & -.22 & Stride length & -.18 & {$[-.31,-.04]$} & $2(200)$ \\
\hline & & & $\underline{\text { Paralanguage }}$ & & & \\
\hline $1(100)$ & & .28 & Ease of understanding & .35 & & $1(100)$ \\
\hline $\mathrm{n} / \mathrm{a}$ & $\mathrm{n} / \mathrm{a}$ & $\mathrm{n} / \mathrm{a}$ & Expressive / varying voice & .01 & {$[-.45, .46]$} & $2(81)$ \\
\hline $3(262)$ & {$[-.07, .35]$} & .15 & Fluent speaking (vs. nervous) & .10 & {$[-.27, .44]$} & $2(200)$ \\
\hline $2(200)$ & {$[-.05, .23]$} & .09 & Pleasantness of voice & .43 & {$[.32, .54]$} & $3(224)$ \\
\hline $2(200)$ & {$[-.22, .18]$} & -.02 & Powerful / confident voice (vs. soft) & .02 & {$[-.11, .16]$} & $3(224)$ \\
\hline $\mathrm{n} / \mathrm{a}$ & $\mathrm{n} / \mathrm{a}$ & $\mathrm{n} / \mathrm{a}$ & Loudness & .18 & {$[-.12, .45]$} & $2(81)$ \\
\hline $2(200)$ & {$[.04, .31]$} & .18 & Pitch & .05 & {$[-.19, .29]$} & $4(699)$ \\
\hline $1(54)$ & & .11 & Speech rate & -.02 & {$[-.28, .26]$} & 2(499) \\
\hline $1(112)$ & & -.11 & Speech vs. non-speech & -.10 & {$[-.35, .16]$} & $2(499)$ \\
\hline $1(138)$ & & -.22 & Interruptions & -.30 & & $1(151)$ \\
\hline & & & Appearance & & & \\
\hline $10(983)$ & {$[.02, .18]$} & .10 & Attractiveness & .32 & {$[.19, .44]$} & 11(897) \\
\hline $6(476)$ & {$[-.08, .16]$} & .04 & Babyfaceness vs. maturity & .33 & {$[.17, .46]$} & $6(467)$ \\
\hline 3(189) & {$[-.23, .06]$} & -.09 & Distinctiveness & .02 & {$[-.12, .17]$} & $3(189)$ \\
\hline $10(882)$ & {$[.03, .24]$} & .13 & Neatness & .18 & {$[.05, .30]$} & $9(658)$ \\
\hline $8(726)$ & {$[-.11, .11]$} & .00 & Stylishness & -.01 & {$[-.11, .09]$} & $6(493)$ \\
\hline $3(212)$ & {$[-.02, .25]$} & .12 & Formality & -.01 & {$[-.15, .13]$} & $3(212)$ \\
\hline $4(249)$ & {$[-.15, .10]$} & -.02 & Eyeglasses & .00 & {$[-.15, .15]$} & $3(176)$ \\
\hline $3(176)$ & {$[-.04, .26]$} & .11 & Volume of Mouth / full lips & .05 & {$[-.13, .22]$} & $3(176)$ \\
\hline $1(100)$ & & .16 & Hair length (long) & .11 & & $1(100)$ \\
\hline $3(176)$ & {$[-.12, .19]$} & .03 & Dark hair color & -.02 & {$[-.17, .13]$} & $3(176)$ \\
\hline $2(200)$ & {$[-.35,-.01]$} & -.18 & Height & -.04 & {$[-.25, .17]$} & $2(200)$ \\
\hline $2(173)$ & {$[-.27, .03]$} & -.12 & Muscular & -.20 & & $1(100)$ \\
\hline $2(200)$ & {$[-.18, .10]$} & -.04 & Weight & .08 & {$[-.06, .22]$} & $2(200)$ \\
\hline $2(173)$ & {$[-.23, .65]$} & .27 & Dark clothes & -.19 & & $1(100)$ \\
\hline
\end{tabular}

Note. $k=$ number of included samples, $n=$ overall sample size. The effect size and confidence interval (CI) estimations are based on a random effects model (empty CI cells included only one study). Cues in bold showed at least small ( $\mathrm{r} \geq .10)$ effects for both utilization and validity (same direction; across at least two studies). 
Table 7

Cue Validity and Cue Utilization Meta-Analyses: Conscientiousness

\begin{tabular}{|c|c|c|c|c|c|c|}
\hline \multicolumn{3}{|c|}{ Cue Validity } & \multirow{2}{*}{$\begin{array}{c}\text { Conscientiousness } \\
\text { Cues }\end{array}$} & \multicolumn{3}{|c|}{ Cue Utilization } \\
\hline$k(n)$ & $95 \% \mathrm{CI}$ & $r$ & & $r$ & $95 \% \mathrm{CI}$ & $k(\mathrm{n})$ \\
\hline & & & Facial expression & & & \\
\hline $5(529)$ & {$[-.04, .22]$} & .09 & Cheerful facial expression & .28 & {$[.10, .44]$} & $7(630)$ \\
\hline $4(414)$ & {$[-.14, .05]$} & -.05 & Dominant facial expression & .13 & {$[-.05, .31]$} & $2(200)$ \\
\hline $\mathrm{n} / \mathrm{a}$ & $\mathrm{n} / \mathrm{a}$ & $\mathrm{n} / \mathrm{a}$ & General expressiveness & $\mathrm{n} / \mathrm{a}$ & $\mathrm{n} / \mathrm{a}$ & $\mathrm{n} / \mathrm{a}$ \\
\hline $2(200)$ & {$[-.10, .18]$} & .04 & Unconcerned (vs. serious) & -.04 & {$[-.24, .17]$} & $2(200)$ \\
\hline $3(363)$ & {$[-.05, .17]$} & .06 & Eye contact & .20 & {$[.04, .34]$} & $4(400)$ \\
\hline $2(116)$ & {$[-.70, .47]$} & -.17 & Eyebrow movements & .15 & {$[-.04, .32]$} & $2(116)$ \\
\hline & & & Body language & & & \\
\hline $3(212)$ & {$[-.27, .32]$} & .03 & Body movement & -.18 & {$[-.36, .00]$} & $5(316)$ \\
\hline $\mathrm{n} / \mathrm{a}$ & $\mathrm{n} / \mathrm{a}$ & $\mathrm{n} / \mathrm{a}$ & Forward lean / proximity & .14 & & $1(60)$ \\
\hline $3(300)$ & {$[-.14, .20]$} & .03 & Gestures & -.01 & {$[-.22, .20]$} & $3(260)$ \\
\hline $2(200)$ & {$[-.30, .29]$} & -.01 & Head movements & -.23 & {$[-.39,-.05]$} & $3(240)$ \\
\hline $3(237)$ & {$[.04, .29]$} & .16 & Self-assured / open vs. slouching & .31 & {$[.14, .46]$} & $2(125)$ \\
\hline $2(213)$ & {$[-.28,-.01]$} & -.15 & Closed arms & -.03 & {$[-.16, .11]$} & $2(213)$ \\
\hline $2(200)$ & {$[-.34,-.07]$} & -.21 & Self-touch & -.22 & {$[-.35,-.09]$} & $2(200)$ \\
\hline $4(325)$ & {$[-.19, .20]$} & .01 & Tension / nervousness (vs. relaxed) & .21 & {$[-.11, .50]$} & $4(325)$ \\
\hline $1(100)$ & & -.16 & Stride length & -.39 & & $1(100)$ \\
\hline & & & Paralanguage & & & \\
\hline $2(200)$ & {$[-.11, .17]$} & .03 & Ease of understanding & .33 & {$[-.05, .63]$} & $2(200)$ \\
\hline $\mathrm{n} / \mathrm{a}$ & $\mathrm{n} / \mathrm{a}$ & $\mathrm{n} / \mathrm{a}$ & Expressive / varying voice & .31 & {$[.10, .50]$} & $2(81)$ \\
\hline $3(338)$ & {$[-.10, .21]$} & .06 & Fluent speaking (vs. nervous) & .27 & {$[.08, .43]$} & $3(351)$ \\
\hline $2(200)$ & {$[-.18, .10]$} & -.04 & Pleasantness of voice & .21 & {$[.02, .39]$} & $3(224)$ \\
\hline $2(200)$ & {$[-.04, .24]$} & .10 & Powerful / confident voice (vs. soft) & .01 & {$[-.13, .15]$} & $3(224)$ \\
\hline $\mathrm{n} / \mathrm{a}$ & $\mathrm{n} / \mathrm{a}$ & $\mathrm{n} / \mathrm{a}$ & Loudness & .08 & {$[-.15, .29]$} & $2(81)$ \\
\hline $1(100)$ & & .04 & Pitch & .14 & {$[-.18, .43]$} & $4(623)$ \\
\hline $1(138)$ & & .02 & Speech rate & .05 & {$[-.25, .34]$} & $3(650)$ \\
\hline $1(112)$ & & .22 & Speech vs. non-speech & .25 & {$[.16, .33]$} & 2(499) \\
\hline $\mathrm{n} / \mathrm{a}$ & $\mathrm{n} / \mathrm{a}$ & $\mathrm{n} / \mathrm{a}$ & Interruptions & $\mathrm{n} / \mathrm{a}$ & $\mathrm{n} / \mathrm{a}$ & $\mathrm{n} / \mathrm{a}$ \\
\hline & & & Appearance & & & \\
\hline $7(606)$ & {$[-.01, .23]$} & .11 & & .21 & {$[.06, .35]$} & $10(875)$ \\
\hline $5(389)$ & {$[-.10, .21]$} & .06 & Babyfaceness vs. maturity & .06 & {$[-.29, .40]$} & $5(445)$ \\
\hline $3(189)$ & {$[-.33,-.05]$} & -.19 & Distinctiveness & -.12 & {$[-.26, .03]$} & $3(189)$ \\
\hline $7(505)$ & {$[.15, .31]$} & .23 & Neatness & .50 & {$[.37, .61]$} & $10(827)$ \\
\hline $6(493)$ & {$[-.01, .17]$} & .08 & Stylishness & .07 & {$[-.11, .25]$} & $6(493)$ \\
\hline $3(212)$ & {$[-.09, .37]$} & .15 & Formality & .54 & {$[.32, .71]$} & $5(445)$ \\
\hline $3(176)$ & {$[-.19, .24]$} & .03 & Eyeglasses & .25 & {$[.10, .38]$} & $3(176)$ \\
\hline $3(176)$ & {$[-.13, .21]$} & .04 & Volume of Mouth / full lips & .04 & {$[-.19, .26]$} & $3(176)$ \\
\hline $2(200)$ & {$[-.30,-.03]$} & -.17 & Hair length (long) & -.14 & {$[-.27, .00]$} & $2(200)$ \\
\hline $3(176)$ & {$[-.12, .18]$} & .03 & Dark hair color & .12 & {$[-.10, .32]$} & $3(176)$ \\
\hline $2(200)$ & {$[-.28,-.01]$} & -.15 & Height & -.06 & {$[-.29, .17]$} & $2(200)$ \\
\hline $1(100)$ & & .00 & Muscular & -.17 & & $1(100)$ \\
\hline $2(200)$ & {$[-.33, .33]$} & .00 & Weight & .06 & {$[-.08, .20]$} & $2(200)$ \\
\hline $1(100)$ & & -.13 & Dark clothes & -.21 & & $1(100)$ \\
\hline
\end{tabular}

Note. $k=$ number of included samples, $n=$ overall sample size. The effect size and confidence interval (CI) estimations are based on a random effects model (empty CI cells included only one study). Cues in bold showed at least small ( $\mathrm{r} \geq .10)$ effects for both utilization and validity (same direction; across at least two studies). 
Table 8

Cue Validity and Cue Utilization Meta-Analyses: Intelligence

\begin{tabular}{|c|c|c|c|c|c|c|}
\hline \multicolumn{3}{|c|}{ Cue Validity } & \multirow{2}{*}{$\frac{\text { Intelligence }}{\text { Cues }}$} & \multicolumn{3}{|c|}{ Cue Utilization } \\
\hline$k(n)$ & $95 \% \mathrm{CI}$ & $r$ & & $r$ & $95 \% \mathrm{CI}$ & $k(\mathrm{n})$ \\
\hline & & & Facial expression & & & \\
\hline $4(267)$ & {$[-.22, .02]$} & -.10 & Cheerful facial expression & .16 & {$[.04, .27]$} & $4(281)$ \\
\hline $1(100)$ & & .34 & Dominant facial expression & .35 & & $1(100)$ \\
\hline $\mathrm{n} / \mathrm{a}$ & $\mathrm{n} / \mathrm{a}$ & $\mathrm{n} / \mathrm{a}$ & General expressiveness & .12 & {$[-.10, .33]$} & $2(122)$ \\
\hline $2(142)$ & {$[-.24, .09]$} & -.08 & Unconcerned (vs. serious) & -.13 & {$[-.61, .41]$} & $2(142)$ \\
\hline $4(267)$ & {$[-.07, .22]$} & .08 & Eye contact & .32 & {$[.19, .43]$} & $3(221)$ \\
\hline \multirow[t]{2}{*}{$\mathrm{n} / \mathrm{a}$} & $\mathrm{n} / \mathrm{a}$ & $\mathrm{n} / \mathrm{a}$ & Eyebrow movements & $\mathrm{n} / \mathrm{a}$ & $\mathrm{n} / \mathrm{a}$ & $\mathrm{n} / \mathrm{a}$ \\
\hline & & & Body language & & & \\
\hline $2(142)$ & {$[-.18, .15]$} & -.01 & Body movement & .03 & {$[-.11, .17]$} & $3(202)$ \\
\hline $3(258)$ & {$[-.01, .24]$} & .12 & Forward lean / proximity & .16 & & $1(42)$ \\
\hline $4(267)$ & {$[-.09, .15]$} & .03 & Gestures & .28 & {$[.11, .43]$} & $4(283)$ \\
\hline $2(88)$ & {$[-.22, .21]$} & -.01 & Head movements & .16 & & $1(42)$ \\
\hline $2(121)$ & {$[-.18, .25]$} & .04 & Self-assured / open vs. slouching & .28 & {$[-.09, .58]$} & $2(121)$ \\
\hline 2(142) & {$[-.16, .18]$} & .01 & Closed arms & -.12 & {$[-.32, .10]$} & $2(142)$ \\
\hline $2(88)$ & {$[-.10, .32]$} & .12 & Self-touch & .06 & {$[-.14, .25]$} & $2(104)$ \\
\hline $3(221)$ & {$[-.39, .20]$} & -.10 & Tension / nervousness (vs. relaxed) & -.15 & {$[-.28,-.02]$} & $3(221)$ \\
\hline \multirow[t]{2}{*}{$1(100)$} & & .17 & Stride length & .20 & & $1(100)$ \\
\hline & & & Paralanguage & & & \\
\hline $4(251)$ & {$[.01, .44]$} & .24 & Ease of understanding & .30 & {$[.12, .46]$} & $4(251)$ \\
\hline $1(42)$ & & -.02 & Expressive / varying voice & .23 & {$[.03, .41]$} & $2(102)$ \\
\hline $3(221)$ & {$[-.28, .44]$} & .09 & Fluent speaking (vs. nervous) & .25 & {$[.07, .42]$} & $6(373)$ \\
\hline $3(221)$ & {$[-.08, .32]$} & .12 & Pleasantness of voice & .22 & {$[.06, .37]$} & $3(221)$ \\
\hline $3(165)$ & {$[-.26, .05]$} & -.11 & Powerful / confident voice (vs. soft) & .18 & {$[.00, .34]$} & $2(135)$ \\
\hline $\mathrm{n} / \mathrm{a}$ & $\mathrm{n} / \mathrm{a}$ & $\mathrm{n} / \mathrm{a}$ & Loudness & .45 & & $1(30)$ \\
\hline 2(142) & {$[-.36,-.04]$} & -.21 & Pitch & .00 & {$[-.25, .25]$} & $2(142)$ \\
\hline 2(109) & {$[-.08, .49]$} & .22 & Speech rate & .31 & {$[.12, .47]$} & $2(109)$ \\
\hline $4(197)$ & {$[-.01, .39]$} & .20 & Speech vs. non-speech & .30 & {$[.13, .45]$} & $3(151)$ \\
\hline \multirow[t]{2}{*}{$\mathrm{n} / \mathrm{a}$} & $\mathrm{n} / \mathrm{a}$ & $\mathrm{n} / \mathrm{a}$ & Interruptions & $\mathrm{n} / \mathrm{a}$ & $\mathrm{n} / \mathrm{a}$ & $\mathrm{n} / \mathrm{a}$ \\
\hline & & & Appearance & & & \\
\hline $3(632)$ & {$[-.26, .24]$} & -.01 & Attractiveness & .53 & {$[.44, .60]$} & $2(602)$ \\
\hline $1(100)$ & & .02 & Babyfaceness vs. maturity & -.10 & & $1(100)$ \\
\hline $1(502)$ & & .00 & Distinctiveness & -.25 & & $1(502)$ \\
\hline $1(100)$ & & -.06 & Neatness & .34 & & $1(100)$ \\
\hline $1(100)$ & & -.11 & Stylishness & .13 & & $1(100)$ \\
\hline $1(100)$ & & -.09 & Formality & .17 & & $1(100)$ \\
\hline $\mathrm{n} / \mathrm{a}$ & $\mathrm{n} / \mathrm{a}$ & $\mathrm{n} / \mathrm{a}$ & Eyeglasses & $\mathrm{n} / \mathrm{a}$ & $\mathrm{n} / \mathrm{a}$ & $\mathrm{n} / \mathrm{a}$ \\
\hline $\mathrm{n} / \mathrm{a}$ & $\mathrm{n} / \mathrm{a}$ & $\mathrm{n} / \mathrm{a}$ & Volume of Mouth / full lips & $\mathrm{n} / \mathrm{a}$ & $\mathrm{n} / \mathrm{a}$ & $\mathrm{n} / \mathrm{a}$ \\
\hline $1(100)$ & & -.05 & Hair length (long) & -.01 & & $1(100)$ \\
\hline $\mathrm{n} / \mathrm{a}$ & $\mathrm{n} / \mathrm{a}$ & $\mathrm{n} / \mathrm{a}$ & Dark hair color & $\mathrm{n} / \mathrm{a}$ & $\mathrm{n} / \mathrm{a}$ & $\mathrm{n} / \mathrm{a}$ \\
\hline $1(100)$ & & .03 & Height & .16 & & $1(100)$ \\
\hline $1(100)$ & & -.07 & Muscular & .20 & & $1(100)$ \\
\hline $1(100)$ & & -.05 & Weight & -.36 & {$[-.50,-.20]$} & $2(130)$ \\
\hline $1(100)$ & & .11 & Dark clothes & .07 & & $1(100)$ \\
\hline
\end{tabular}

Note. $k=$ number of included samples, $n=$ overall sample size. The effect size and confidence interval (CI) estimations are based on a random effects model (empty CI cells included only one study). Cues in bold showed at least small ( $\mathrm{r} \geq .10)$ effects for both utilization and validity (same direction; across at least two studies). 


\section{Overview results}

In the following, we will summarize the results separately for each trait domain. We hereby focus on cue validities and cue utilizations that were at least small in effect size $(r \geq$ .10; Cohen, 1992). Cues that were used by observers to judge a specific trait and were at the same time related to actual trait values (in the same direction, with results from at least two studies), were identified as potential influences that drive trait accuracies.

Neuroticism. Non-cheerful expressions, a tense and nervous body language, an unexpressive, non-fluent, unpleasant, and silent voice, low amount of speaking, unattractiveness and a non-neat appearance, low height and being less muscular were all indicators of being neurotic and led observers to the same impression. Other cues (e.g., dominant facial expression, self-assured body language, ease of understanding, powerful voice, not wearing eyeglasses) were associated with being judged less neurotic but were not related to individuals' actual neuroticism. Wearing darker clothes and having darker hair were one of the few cues that were related to actual levels of neuroticism but were not used by observers when judging this trait (see Table 3).

Extraversion. Out of all the investigated traits, extraversion included the greatest number of valid cues that were also used by observers. These cues include cheerful facial expression, dominant facial expression, general facial expressiveness, forward leans, gestures, self-assured posture, relaxed posture, expressive/varying voice, fluent speaking, pleasantness of voice, confidence of voice, loudness of voice, speech rate, amount of talking, attractiveness, neatness, stylishness, lack of eyeglasses, volume of mouth, hair length, and the lack of dark clothes. The cues eye contact, body movement, and head movements (among others) were used by observers to judge extraversion but were not related to actual extraversion (see Table 4).

Openness. For openness, only self-assured/open posture, pleasantness of voice, volume of mouth, and hair length were valid and utilized cues. There were multiple cues (e.g., 
cheerful facial expression, fluent speaking, attractiveness, neatness, stylishness) that were used when judging openness, however, they were not related to actual openness. Less talking was related to actual openness, but observers did not use this cue (see Table 5).

Agreeableness. A cheerful facial expression, small stride length, fluent speaking, attractiveness, and neatness of appearance were the only cues that were related to actual agreeableness and used by observers to judge this trait. Many cues that observers associated with agreeableness (e.g., eye contact, relaxed body language, pleasantness of voice, babyfaceness) were not valid indicators. Furthermore, there were a few valid cues (high pitch, non-dominant facial expression) that were not identified by observers (see Table 6).

Conscientiousness. Cues that drove accuracy for conscientiousness judgments exclusively belonged to the domains of body language and appearance. These were selfassured/open posture, lack of self-touch, attractiveness, lack of distinctiveness in appearance, neatness, formality, and having shorter hair. Many more cues (e.g., fluent speaking, pleasantness of voice, wearing eyeglasses) were used when judging conscientiousness, but were not actually related with individuals' conscientiousness (see Table 7).

Intelligence. Here, cue validities refer to the relationship between cues and intelligence measured via cognitive ability tests (with the exception of one study that only included self-reports). For intelligence, paralinguistic cues played the biggest role in explaining accuracy. Especially, easiness of understanding, pleasantness of voice, speech rate, and amount of speech were used by observers and at the same time related to actual (measured) intelligence. Cues that were used but not valid were (for example): eye contact, amount of gestures, powerful voice, and attractiveness ${ }^{2}$ (see Table 8).

\section{Summary and comparison.}

Results of the included studies suggest that, for all traits, there are at least a few nonverbal cues that allow for accurate glimpses into one's personality. For most traits (i.e., extraversion, openness, conscientiousness, intelligence) this is in line with research showing 
above chance judgment accuracies, even by strangers (for overviews see: Connelly \& Ones, 2010; Connolly, Kavanagh, \& Viswesvaran, 2007; Hall, Andrzejewski, Murphy, Mast, \& Feinstein, 2008; Kenny et al., 1992; Kenny, Albright, Malloy, \& Kashy, 1994). However, even for traits that are typically viewed as not easily observable (i.e., agreeableness, neuroticism) we find multiple valid cues.

Nearly all the valid cues were also used by observers when judging the specific traits, showing that perceivers generally have the ability to correctly identify relevant cues. However, observers often overestimated the actual size of relationships, this potentially resulted in lower accuracies. Furthermore, there were many cues for which the included studies did not provide evidence for validity, but that were nevertheless used for judgments. These cues, thus, might represent some sort of common bias/inaccurate stereotype when judging the respective traits.

The five most utilized cues across all traits were attractiveness, dominant facial expression, cheerful facial expression, pleasantness of voice, and ease of understanding $(r=$ .33 - .45). The five most valid cues were speech vs. non-speech, loudness, expressive / varying voice, wearing dark clothes, and speech rate $(r=.14-.18)$. Generally, the portion of cues that drove accuracy was highest for the paralanguage domain, highlighting the importance of voice and speech characteristics when judging personality.

In Table S3 in the online supplement, we additionally provide vector correlations ${ }^{3}$ for cue validities and cue utilizations across traits. Results show high inter-correlations for cue utilizations. This especially applies to extraversion, neuroticism (recoded as emotional stability), and intelligence (mean intercorrelations for the three traits $=.68$ ), as well as to openness and agreeableness $(r=.72)$. This means that for judgments on these traits, available cues were used in a similar way (i.e., in the sense that the most and least utilized cues for one trait were also the most and least used cues for the other traits). The strong relationships between trait judgments might be related to general favorability of these traits and specific 
cues (e.g., attractive individuals, with a cheerful expression and self-assured postures were generally judged as being high in extraversion, emotional stability, and intelligence). Low correlations were found for the cue utilization regarding extraversion and conscientiousness $(r$ $=.14)$.

For cue validities, we found a more mixed pattern: There were high inter-correlations between the cue validities for extraversion, emotional stability, and conscientiousness (mean intercorrelations for the three traits $r=.51$ ). For example, the neatness of appearance was significantly correlated with self/informant ratings on all three traits, while voice pitch played a negligible role. Noticeably smaller or even negative (e.g., openness $\&$ conscientiousness: $r$ $=-.27$ ) correlations between cue validities were found for other trait combinations. This suggests that the relationship between nonverbal cues and actual personality across traits is more diverse than the perceived associations.

\section{Potential Moderators}

The mentioned relationship between nonverbal cues and personality (judgments) can be influenced by a variety of personal, situational, and trait-specific factors that affect the strength of cue utilities/validities. Funder (1999, 2012, also see Ch. 2 by Letzring \& Funder in this handbook) distinguishes between four classes of moderators: Differences between traits (good trait), differences regarding the available information (good information), differences between perceivers (good judge), and differences between targets (good target).

A good trait on the individual cue level refers to traits that have, relative to other traits, a high number of valid cues that are also utilized by observers. Comparing the traits, one can notice that extraversion stands out by being associated with the highest number of valid and utilized nonverbal cues. This is in line with previous research identifying extraversion as a trait that is easily-observable across many different contexts (see Back \& Nestler, 2016 and overviews mentioned above). Agreeableness however, is one of the traits hardest to judge accurately. Our results show that the lack of accuracy when judging agreeableness is not 
generally due to missing knowledge or judgment biases but also due to the limited number of valid cues available (e.g., for agreeableness only $10 \%$ of investigated cues showed validities of at least $r=.20$. Extraversion in contrast had a percentage of 18).

The quantity and quality of information (i.e., good information) can also influence cue validities and cue utilities. One could imagine, for example, that over time (of interacting with someone) cue validities for dynamic cues increase due to more reliable variance in cue expression between different targets (e.g., reliable differences in the amount of head movements, which could be related to extraversion, might only emerge after a few minutes of interaction). Furthermore, the type of situation and their trait relevance might serve as moderators for cue validities (e.g., a tense body language might be a highly valid cue for judging neuroticism when the situation activates this specific trait but not in a neutral situation). While levels of accuracy have shown to increase over time (e.g., Borkenau et al., 2004; Letzring, Wells, \& Funder, 2006) and to differ between settings varying in trait relevance (Hirschmüller, Egloff, Schmukle, Nestler, \& Back, 2015; Letzring et al., 2006), influences on single cue validities have not been investigated yet. Considering the right side of the lens model (i.e., cue utilities), good information can also refer to the type of judgment channel (e.g., video with or without sound, audio only) observers are exposed to. For example, multiple studies included in this chapter (e.g., Borkenau \& Liebler, 1992a, 1992b, 1995; Murphy, Hall, \& Colvin, 2003; Reynolds \& Gifford, 2001) compare cue utilizations for different kinds of judgments channels. Results by Borkenau and Liebler (1995) suggest that if valid cues are not observable (e.g., there are no paralinguistic cues), the utilization of observable non-valid cues (e.g., specific body language cues) increases, which would result in lower accuracy.

Regarding the expression nonverbal cues, good targets would refer to a group of individuals that provides more nonverbal cues and/or more variance in nonverbal cues, resulting in potentially stronger cue validities. Generally, research has identified the traits of 
extraversion and emotional stability as features of good targets (i.e., targets that act more and express their emotions, cf. Colvin, 1993; Human \& Biesanz, 2011; Human, Biesanz, Finseth, Pierce, \& Le, 2014), but, to the best of our knowledge, specific changes in cue validities or utilities have not been investigated. There have, however, been many studies separating results by targets' sex (e.g., Aronovitch, 1976; Berry \& Landry, 1997; Lippa, 1998; Riggio \& Friedman, 1986; Shrout \& Fiske, 1981; Simpson, Gangestad, \& Biek, 1993). Lippa (1998), for example, found multiple nonverbal cues that were valid indicators of females' extraversion but not of males' extraversion, suggesting that it should be easier to judge females' extraversion.

Someone who is identified as a good judge should be better at identifying and using valid cues (and not using non-valid cues) compared to other judges, thus reaching higher accuracies. Another influence could be that good judges evoke more valid cues in targets. As most judgment accuracy studies do not include interactions between observers and targets, this explanation has received little empirical attention so far (but see Letzring, 2008). While the question "who is a good judge" has engaged researchers for a long time, results have been ambiguous (cf. Back \& Nestler, 2016, see Davis \& Kraus, 1997; Hall, Andrzejewski, \& Yopchick, 2009; Taft, 1955 for meta-analyses). There have been a few studies analyzing cue utilities for different groups of (potentially good) judges (e.g., Hartung \& Renner, 2011; Hirschmüller, Egloff, Nestler, \& Back, 2013; Nestler \& Back, 2017). For example, Hartung and Renner (2011) showed that socially highly curious judges generally used more available cues and were thus more likely to detect valid cues for visible traits (in this case extraversion and openness).

\section{Outlook and future research}

Here we will summarize suggestions that, in our view, would benefit future research on nonverbal cues and their relation to trait accuracy. First, while meta-analytical results presented in this chapter can serve as a first overview of validities and utilities for a variety of 
cues, more, and more comprehensive studies are needed. Most included studies only investigated a small number of cues $(<10)$ and there are many trait-cue combinations for which empirical evidence is limited. It would also be beneficial to replicate the presented results across more diverse target samples and judgment contexts. Furthermore, results across studies are often difficult to integrate. For example, some studies only presented correlations controlled for sex and age, while other studies only reported significant cue relationships. Furthermore, nearly all studies focused on aggregated observer utilities (i.e., correlations computed for personality judgments aggregated across observers). Results based on this approach, in contrast to the single observer approach (separate correlations for each observer), depend on the number of observers within a study (more observers lead to higher utilities because of higher reliability of those ratings, cf. Back \& Nestler, 2016; Hall \& Bernieri, 2001; Nestler \& Back, 2017), making it difficult to compare studies with a varying number of observers. Thus, we urge researchers, to (at least in online supplementaries) present comprehensive results, which include zero-order cue validities and cue utilities (aggregated and single observer) for all assessed cue and trait combinations.

Second, as stated previously, the research on how potential moderators influence cue validities and cue utilities, especially good information, good target, and good judge, is limited. Therefore, it would be beneficial to investigate how cue validities change over time and settings (good information) and to additionally present results separated by specific target criteria (besides sex), to identify subgroups that might constitute as good targets. In a similar way, systematically comparing single-observer cue utilities across judges would shed more light on the good judge.

Third, the advance of big data and accompanying machine learning techniques provides opportunities for the investigation of nonverbal cues. Research shows that, based on digital footprints, computer algorithms are often better in judging personality than humans (Youyou, Kosinski, \& Stillwell, 2015) and it would be fruitful to transfer these methods to the 
judgment of pictures, videos, or audio files. Thus, it could be investigated if computer algorithms can outperform human judges in these contexts and how humans vs. computers differ in their cue utilizations. Machine learning approaches could also be used as an economic and more objective tool for the quantification of nonverbal cues (i.e., use machine learning approaches instead of raters to judge the degree of smiling of targets).

In summary, with this chapter, we offer a first overview of how and which nonverbal cues contribute to accuracy for different traits. Results show that, for all traits, there are at least a few nonverbal cues that allow for accurate glimpses into one's personality, and perceivers often showed the ability to identify relevant cues. We plan to expand this overview in the future and to regularly update the results in the online supplement (osf.io/9p64g). We hereby invite interested readers to contribute to a more exhaustive documentation and add additional studies. In the years to come, with collaborations like this, we will be able to offer more comprehensive insights on which cues drive trait accuracies.

\section{Footnotes}

${ }^{1}$ With the raw data provided on osf.io/9p64g it is easily possible to compute results for different kinds of allocations (e.g., separate results for dominance and sociability aspects of extraversion; separate results for stylishness related to hairstyles).

${ }^{2}$ For more studies and meta-analyses concerning the specific link between attractiveness and intelligence please refer to Jackson, Hunter, \& Hodge, 1995, Kanazawa, 2011, Langlois et al., 2000, and Mitchem et al., 2015. Mirroring the results we present here, recent research suggests that there is an absent or low correlation between attractiveness and actual intelligence (cf. Mitchem et al., 2015).

${ }^{3}$ In this case vector correlations refer to correlations between Fisher z-transformed cue validity/utilization correlations across traits. 
Table S1.

Studies Investigating the Relationship Between Nonverbal Cues and Personality / Personality

Judgments

\begin{tabular}{|c|c|c|c|c|c|c|c|c|}
\hline Authors & Type & $\begin{array}{l}N \\
(\mathbf{T})\end{array}$ & $\begin{array}{l}N: \\
(\mathbf{P})\end{array}$ & $\begin{array}{l}\text { Jud. } \\
\text { Chan } \\
\text { nel } \\
\end{array}$ & $\begin{array}{l}N: \\
(\mathbf{R})\end{array}$ & $\begin{array}{l}\text { Cue } \\
\text { Types }\end{array}$ & $\begin{array}{c}\text { No. } \\
\text { of } \\
\text { cues }\end{array}$ & Traits \\
\hline $\begin{array}{l}\text { Albright, Kenny, \& Malloy } \\
\text { (1988; Study 3) }\end{array}$ & $\mathrm{CU}$ & 169 & $3-5$ & Inter & $\mathrm{P}$ & Ap & 5 & E O C \\
\hline $\begin{array}{l}\text { Albright, Malloy, Dong, } \\
\text { Kenny, Fang, Winquist, \& } \\
\text { Yu (1997; Study 1) }\end{array}$ & $\mathrm{CU}$ & 80 & 4 & Inter & $\mathrm{P}$ & Fe Ap & 4 & NEOAC \\
\hline $\begin{array}{l}\text { Albright, Malloy, Dong, } \\
\text { Kenny, Fang, Winquist, \& } \\
\text { Yu (1997; Study 2) }\end{array}$ & $\mathrm{CU}$ & 149 & $4-5$ & $\mathrm{Ph}$ & $\mathrm{P}$ & Fe Ap & 4 & NE O A C \\
\hline Aronovitch (1976) & $\mathrm{CU}$ & 57 & 50 & $\mathrm{Au}$ & obj & $\mathrm{Pl}$ & 6 & N E A C \\
\hline $\begin{array}{l}\text { Asendorpf, Banse, \& Mücke } \\
\text { (2002) }\end{array}$ & $\begin{array}{l}\mathrm{CV} \\
\mathrm{CU}\end{array}$ & 139 & 3 & VS & $1-2$ & $\begin{array}{c}\mathrm{Fe} \mathrm{Bl} \\
\mathrm{Pl}\end{array}$ & 6 & $\mathrm{E}$ \\
\hline $\begin{array}{l}\text { Back, Penke, Schmukle, } \\
\text { Sachse, Borkenau, \& } \\
\text { Asendorpf (2011) }\end{array}$ & $\mathrm{CV}$ & 382 & & & 30 & Ap & 1 & $\mathrm{E}$ \\
\hline $\begin{array}{l}\text { Back, Schmukle, \& Egloff } \\
\text { (2010) \& (2011) }\end{array}$ & $\mathrm{CV}$ & 73 & & & 4 & $\begin{array}{l}\text { Fe Bl } \\
\text { Pl Ap }\end{array}$ & 22 & E A \\
\hline Berry \& Hansen (2000) & $\mathrm{CV}$ & 112 & & & 2 & $\begin{array}{c}\mathrm{Fe} \mathrm{Bl} \\
\mathrm{Pl}\end{array}$ & 8 & NEOAC \\
\hline Berry \& Landry (1997) & $\mathrm{CV}$ & 113 & & & $\begin{array}{l}14- \\
22\end{array}$ & Ap & 2 & NEOAC \\
\hline $\begin{array}{l}\text { Biel, Aran, \& Gatica-Perez } \\
\text { (2011) }\end{array}$ & $\mathrm{CU}$ & 442 & 5 & VS & obj & $\mathrm{B} 1 \mathrm{Pl}$ & 11 & NEOAC \\
\hline $\begin{array}{l}\text { Borkenau, Brecke, Möttig, \& } \\
\text { Paelecke (2009) }\end{array}$ & $\begin{array}{l}\mathrm{CU} \\
\mathrm{CV}\end{array}$ & 149 & 24 & $\mathrm{Ph}$ & 7 & $\mathrm{Fe}$ & 1 & NEOA C \\
\hline $\begin{array}{l}\text { Borkenau \& Liebler (1992a) } \\
\& \text { (1992b) }\end{array}$ & $\begin{array}{l}\mathrm{CU} \\
\mathrm{CV}\end{array}$ & 100 & 6 & $\begin{array}{l}\mathrm{VS} \\
\mathrm{VwS} \\
\mathrm{Au}\end{array}$ & $\mathrm{P}$ & $\begin{array}{l}\text { Fe Bl } \\
\text { Pl Ap }\end{array}$ & 45 & NEOAC \\
\hline Borkenau \& Liebler (1995) & $\begin{array}{l}\mathrm{CV} \\
\mathrm{CU}\end{array}$ & 100 & 6 & $\begin{array}{l}\text { VS } \\
\text { Vws }\end{array}$ & $\begin{array}{l}2-6 \\
\text { obj }\end{array}$ & $\begin{array}{l}\text { Fe Bl } \\
\text { Pl Ap }\end{array}$ & 59 & NEO A C I \\
\hline $\begin{array}{l}\text { Brown, Palameta, \& Moore } \\
(2003)^{\mathrm{a}}\end{array}$ & $\begin{array}{l}\mathrm{CV} \\
\mathrm{CU}\end{array}$ & 20 & 30 & VS & 2 & $\begin{array}{c}\text { Fe Bl } \\
\text { Ap }\end{array}$ & 7 & $\mathrm{~A}$ \\
\hline $\begin{array}{l}\text { Burgoon, Birk, \& Pfau } \\
\text { (1990) }\end{array}$ & $\mathrm{CU}$ & 60 & 30 & Inter & 2 & $\begin{array}{l}\mathrm{Fe} \mathrm{Bl} \\
\mathrm{Pl}\end{array}$ & 9 & N E I \\
\hline Burnett \& Motowidlo (1998) & $\mathrm{CU}$ & 60 & $5-8$ & Vws & $2-5$ & $\begin{array}{c}\mathrm{Fe} \mathrm{Bl} \\
\mathrm{Ap}\end{array}$ & 5 & E A C \\
\hline
\end{tabular}




\begin{tabular}{|c|c|c|c|c|c|c|c|c|}
\hline Authors & Type & $\begin{array}{l}N \\
(\mathbf{T})\end{array}$ & $\begin{array}{l}N: \\
(\mathbf{P})\end{array}$ & $\begin{array}{l}\text { Jud. } \\
\text { Chan } \\
\text { nel } \\
\end{array}$ & $\begin{array}{l}N: \\
(\mathbf{R})\end{array}$ & $\begin{array}{l}\text { Cue } \\
\text { Types }\end{array}$ & $\begin{array}{c}\text { No. } \\
\text { of } \\
\text { cues }\end{array}$ & Traits \\
\hline Campbell \& Rushton (1978) & $\mathrm{CV}$ & 46 & & & $1-2$ & $\begin{array}{l}\mathrm{Fe} \mathrm{Bl} \\
\mathrm{Pl}\end{array}$ & 15 & NE I \\
\hline Creed \& Funder (1997) & $\mathrm{CV}$ & 149 & & & 4 & $\mathrm{Bl} \mathrm{Pl}$ & 5 & $\mathrm{~N}$ \\
\hline Eaton \& Funder (2003) & $\begin{array}{l}\mathrm{CV} \\
\mathrm{CU}\end{array}$ & 152 & 1 & Inter & 4 & $\mathrm{Pl}$ & 2 & $\mathrm{E}$ \\
\hline Ferguson (1977) & $\begin{array}{l}\mathrm{CV} \\
\mathrm{CU}\end{array}$ & 15 & 1 & Inter & $?$ & $\mathrm{Pl}$ & 4 & $\mathrm{E}$ \\
\hline Funder \& Sneed (1993) & $\begin{array}{l}\mathrm{CV} \\
\mathrm{CU}\end{array}$ & 151 & 2 & VS & 6 & $\begin{array}{c}\mathrm{Fe} P l \\
\mathrm{Bl}\end{array}$ & 9 & NEOA C \\
\hline $\begin{array}{l}\text { Gifford \& O'Connor (1987; } \\
\text { Study 2) \& Gifford (1991) \& } \\
\text { (1994) }\end{array}$ & $\begin{array}{l}\mathrm{CV} \\
\mathrm{CU}\end{array}$ & 60 & 21 & Vws & $1-2$ & $\mathrm{Fe} \mathrm{Bl}$ & 29 & E A \\
\hline $\begin{array}{l}\text { Gifford, Ng, \& Wilkinson } \\
\text { (1985) }\end{array}$ & $\begin{array}{l}\mathrm{CV} \\
\mathrm{CU}\end{array}$ & 34 & 18 & Vws & 2 & $\begin{array}{l}\text { Fe Bl } \\
\text { Pl Ap }\end{array}$ & 12 & $\begin{array}{c}\text { Other } \\
\text { (social skills, } \\
\text { motivation) }\end{array}$ \\
\hline $\begin{array}{l}\text { Gillath, Bahns, Ge \& } \\
\text { Crandall (2012) }\end{array}$ & $\begin{array}{l}\mathrm{CV} \\
\mathrm{CU}\end{array}$ & 208 & 3 & $\begin{array}{c}\mathrm{PH} \\
\text { (shoes) }\end{array}$ & 2 & Ap & 18 & NE O A C \\
\hline Hartung \& Renner (2011) & $\begin{array}{l}\mathrm{CV} \\
\mathrm{CU}\end{array}$ & 182 & 1 & Inter & $\mathrm{P}$ & $\begin{array}{l}\text { Fe Bl } \\
\text { Pl Ap }\end{array}$ & 62 & E O \\
\hline $\begin{array}{l}\text { Hirschmüller, Egloff, Nestler } \\
\text { \& Back (2013): Study } 1 \text { \& } 2\end{array}$ & $\begin{array}{l}\mathrm{CV} \\
\mathrm{CU}\end{array}$ & 56 & 177 & VS & $2-6$ & Pl Ap & 3 & $\mathrm{E}$ \\
\hline $\begin{array}{l}\text { Hirschmüller, Egloff, } \\
\text { Schmuckle, Nestler, \& Back } \\
\text { (2015) }\end{array}$ & $\begin{array}{l}\mathrm{CV} \\
\mathrm{CU}\end{array}$ & 50 & 45 & VS & $4-6$ & Pl & 1 & $\mathrm{~N}$ \\
\hline $\begin{array}{l}\text { Hirschmüller, Schmukle, } \\
\text { Krause, Back \& Egloff } \\
\text { (2018) }\end{array}$ & $\begin{array}{l}\mathrm{CV} \\
\mathrm{CU}\end{array}$ & 99 & 40 & VS & 4 & $\begin{array}{l}\text { Fe Bl } \\
\text { Pl Ap }\end{array}$ & 26 & $\mathrm{~N}$ \\
\hline Ickes \& Barnes (1977) & $\mathrm{CV}$ & 124 & & & 2 & $\mathrm{Pl}$ & 1 & $\mathrm{~N}$ \\
\hline $\begin{array}{l}\text { Kaurin, Heil, Wessa, Egloff, } \\
\text { \& Hirschmüller (2018) }\end{array}$ & $\begin{array}{l}\mathrm{CV} \\
\mathrm{CU}\end{array}$ & 104 & 15 & VS & 2 & $\begin{array}{c}\mathrm{Fe} \mathrm{Bl} \\
\mathrm{Ap}\end{array}$ & 18 & NEO A C \\
\hline Kendon, \& Cook (1969) & $\mathrm{CV}$ & 15 & & & 1 & $\mathrm{Fe} \mathrm{Pl}$ & 5 & NEA \\
\hline $\begin{array}{l}\text { Kenny, Horner, Kashy, \& } \\
\text { Chu (1992): Study } 1\end{array}$ & $\mathrm{CU}$ & 64 & 56 & VS & 4 & $\begin{array}{c}\mathrm{Fe} \mathrm{Bl} \\
\mathrm{Ap}\end{array}$ & 4 & NEOAC \\
\hline $\begin{array}{l}\text { Kenny, Horner, Kashy, \& } \\
\text { Chu (1992): Study } 2\end{array}$ & $\mathrm{CU}$ & 108 & 3 & inter & $\mathrm{P}$ & Ap & 1 & $\mathrm{E}$ \\
\hline $\begin{array}{l}\text { Kenny, Horner, Kashy, \& } \\
\text { Chu (1992): Study } 3\end{array}$ & $\mathrm{CU}$ & $\begin{array}{r}70 / \\
83\end{array}$ & $3-4$ & inter & $\mathrm{P}$ & Ap & 1 & $\mathrm{E}$ \\
\hline
\end{tabular}




\begin{tabular}{|c|c|c|c|c|c|c|c|c|}
\hline Authors & Type & $\begin{array}{l}N \\
(\mathbf{T})\end{array}$ & $\begin{array}{l}N: \\
(\mathbf{P})\end{array}$ & $\begin{array}{l}\text { Jud. } \\
\text { Chan } \\
\text { nel } \\
\end{array}$ & $\begin{array}{l}N: \\
(\mathbf{R})\end{array}$ & $\begin{array}{l}\text { Cue } \\
\text { Types }\end{array}$ & $\begin{array}{c}\text { No. } \\
\text { of } \\
\text { cues }\end{array}$ & Traits \\
\hline $\begin{array}{l}\text { Koppensteiner \& Grammer } \\
\text { (2010) }\end{array}$ & $\mathrm{CU}$ & 40 & 30 & $\begin{array}{l}\text { Vws } \\
\text { (stick- } \\
\text { figure) }\end{array}$ & obj & $\mathrm{B} 1$ & 8 & NEOA C \\
\hline $\begin{array}{l}\text { Koppensteiner, Stephan \& } \\
\text { Jäschke (2016) }\end{array}$ & $\mathrm{CU}$ & 60 & $\begin{array}{r}18- \\
22\end{array}$ & $\begin{array}{l}\text { Vws } \\
\text { (stick- } \\
\text { figure) }\end{array}$ & obj & B1 & 7 & E A I \\
\hline Levesque \& Kenny (1993) & $\mathrm{CV}$ & 80 & & & $1-2$ & $\mathrm{Bl} \mathrm{Pl}$ & 4 & $\mathrm{E}$ \\
\hline Lippa (1998) & $\begin{array}{l}\mathrm{CV} \\
\mathrm{CU}\end{array}$ & 67 & 6 & VS & 2 & $\begin{array}{l}\mathrm{Fe} \mathrm{Bl} \\
\mathrm{Pl}\end{array}$ & 28 & $\mathrm{E}$ \\
\hline $\begin{array}{l}\text { Lyons, Tickle-Degnen, } \\
\text { Henry, Cohn (2004) }\end{array}$ & $\begin{array}{l}\mathrm{CV} \\
\mathrm{CU}\end{array}$ & 12 & 33 & VS & 4 & $\begin{array}{l}\text { Fe Bl } \\
\text { Pl Ap }\end{array}$ & 9 & NEOA C \\
\hline Mallory \& Miller (1958) & $\mathrm{CV}$ & 372 & & & $?$ & Pl & 5 & $\mathrm{E}$ \\
\hline $\begin{array}{l}\text { Meier, Robinson, Carter, \& } \\
\text { Hinsz (2010): Study } 1\end{array}$ & $\mathrm{CV}$ & 84 & & & $\begin{array}{l}10- \\
18\end{array}$ & Fe Ap & 3 & E A \\
\hline $\begin{array}{l}\text { Meier, Robinson, Carter, \& } \\
\text { Hinsz (2010): Study } 2\end{array}$ & $\mathrm{CV}$ & 133 & & & $\begin{array}{c}10- \\
18\end{array}$ & Fe Ap & 3 & E A \\
\hline Montepare \& Dobish (2003) & $\mathrm{CU}$ & 32 & $\underset{12}{\approx}$ & $\mathrm{Ph}$ & 8 & $\mathrm{Fe}$ & 5 & E A \\
\hline Murphy (2007) & $\begin{array}{l}\mathrm{CU} \\
\mathrm{CV}\end{array}$ & 42 & $\begin{array}{l}7 / \\
20\end{array}$ & VS & $1-2$ & $\begin{array}{l}\mathrm{Fe} \mathrm{Bl} \\
\mathrm{Pl}\end{array}$ & 26 & I \\
\hline $\begin{array}{l}\text { Murphy, Hall, \& Colvin } \\
\text { (2003) }\end{array}$ & $\begin{array}{l}\mathrm{CV} \\
\mathrm{CU}\end{array}$ & 79 & $\underset{35}{\approx}$ & VS & 5 & $\begin{array}{l}\mathrm{Fe} \mathrm{Bl} \\
\mathrm{Pl}\end{array}$ & 17 & I \\
\hline $\begin{array}{l}\text { Naumann, Vazire, Rentfrow, } \\
\& \text { Gosling (2009) }\end{array}$ & $\begin{array}{l}\mathrm{CV} \\
\mathrm{CU}\end{array}$ & 113 & 6 & $\mathrm{Ph}$ & 2 & $\begin{array}{l}\mathrm{Fe} \mathrm{B1} \\
\mathrm{Ap}\end{array}$ & 10 & NEOAC \\
\hline $\begin{array}{l}\text { Nestler, Egloff, Küfner, \& } \\
\text { Back (2012): Study } 1\end{array}$ & $\begin{array}{l}\mathrm{CV} \\
\mathrm{CU}\end{array}$ & 36 & 91 & $\mathrm{Ph}$ & $\begin{array}{c}3 \\
\text { obj }\end{array}$ & Ap & 11 & NEOA C \\
\hline $\begin{array}{l}\text { Nestler, Egloff, Küfner, \& } \\
\text { Back (2012): Study } 2\end{array}$ & $\begin{array}{l}\mathrm{CV} \\
\mathrm{CU}\end{array}$ & 40 & 95 & $\mathrm{PH}$ & $\begin{array}{c}3 \\
\text { obj }\end{array}$ & Ap & 12 & NEOA C \\
\hline Pedersen (1973) & $\mathrm{CV}$ & 170 & & & obj & B1 & 1 & N E I \\
\hline Penke \& Asendorpf (2008) & $\mathrm{CV}$ & 283 & & & 2 & $\mathrm{Fe} \mathrm{Bl}$ & 4 & $\begin{array}{c}\text { Other } \\
\text { (sociosexuality) }\end{array}$ \\
\hline $\begin{array}{l}\text { Petrician, Todorov \& Grady } \\
\text { (2014) }\end{array}$ & $\mathrm{CV}$ & 102 & & & obj & $\mathrm{Fe}$ & 3 & NEOA C \\
\hline Reynolds \& Gifford (2001) & $\begin{array}{l}\mathrm{CV} \\
\mathrm{CU}\end{array}$ & 30 & $\begin{array}{l}7- \\
28\end{array}$ & $\begin{array}{l}\text { VS Au } \\
\text { Vws }\end{array}$ & $\begin{array}{l}2- \\
57\end{array}$ & Pl Ap & 10 & I \\
\hline Riggio \& Friedman (1986) & $\begin{array}{l}\mathrm{CV} \\
\mathrm{CU}\end{array}$ & 62 & 12 & VS & $2-5$ & $\begin{array}{c}\mathrm{Fe} \mathrm{Bl} \\
\mathrm{Pl}\end{array}$ & 5 & NE A I \\
\hline $\begin{array}{l}\text { Riggio, Lippa, \& Salinas } \\
\text { (1990) }\end{array}$ & $\begin{array}{l}\mathrm{CV} \\
\mathrm{CU}\end{array}$ & $\begin{array}{r}28- \\
54\end{array}$ & 12 & VS & $2-5$ & $\mathrm{Fe} \mathrm{Bl}$ & 5 & NE A \\
\hline
\end{tabular}




\begin{tabular}{|c|c|c|c|c|c|c|c|c|}
\hline Authors & Type & $\begin{array}{l}N \\
(\mathbf{T})\end{array}$ & $\begin{array}{l}N: \\
(\mathbf{P})\end{array}$ & $\begin{array}{l}\text { Jud. } \\
\text { Chan } \\
\text { nel } \\
\end{array}$ & $\begin{array}{l}N: \\
(\mathbf{R})\end{array}$ & $\begin{array}{l}\text { Cue } \\
\text { Types }\end{array}$ & $\begin{array}{c}\text { No. } \\
\text { of } \\
\text { cues }\end{array}$ & Traits \\
\hline Scherer (1978) & $\begin{array}{l}\mathrm{CV} \\
\mathrm{CU}\end{array}$ & 24 & 9 & $\mathrm{Au}$ & $\begin{array}{l}6- \\
10\end{array}$ & $\mathrm{Pl}$ & 12 & NE A C \\
\hline Schmid Mast \& Hall (2004) & $\begin{array}{l}\mathrm{CV} \\
\mathrm{CU}\end{array}$ & 48 & 5 & $\mathrm{Ph}$ & $2-5$ & $\begin{array}{l}\text { Fe Bl } \\
\text { Ap }\end{array}$ & 10 & Other (status) \\
\hline $\begin{array}{l}\text { Schultheiss \& Brunstein, } \\
\text { (2002) }\end{array}$ & $\begin{array}{l}\mathrm{CV} \\
\mathrm{CU}\end{array}$ & 68 & 4 & VS & 2 & $\begin{array}{l}\text { Fe Bl } \\
\text { Pl }\end{array}$ & 13 & $\mathrm{E}$ \\
\hline Shrout \& Fiske (1981) & $\mathrm{CU}$ & 44 & 8 & VS & $>1$ & $\begin{array}{l}\mathrm{Fe} \mathrm{Bl} \\
\mathrm{Pl}\end{array}$ & 14 & A \\
\hline $\begin{array}{l}\text { Simpson, Gangestad, \& Biek } \\
\text { (1993) }\end{array}$ & $\begin{array}{l}\mathrm{CU} \\
\mathrm{CV}\end{array}$ & 210 & $>1$ & Vws & $>1$ & $\mathrm{Fe} \mathrm{Bl}$ & 17 & E A O \\
\hline $\begin{array}{l}\text { Stopfer, Egloff, Nestler, \& } \\
\text { Back (2014) }\end{array}$ & $\mathrm{CU}$ & $\begin{array}{c}93- \\
103 \\
\end{array}$ & 12 & $\mathrm{Ph}$ & $2-6$ & Fe Ap & 3 & NE O A C \\
\hline $\begin{array}{l}\text { ten Brinke, Porter, Korva, } \\
\text { Fowler, Lilienfeld, \& Patrick } \\
\text { (2017) }\end{array}$ & $\begin{array}{l}\mathrm{CU} \\
\mathrm{CV}\end{array}$ & $\begin{array}{r}50- \\
51\end{array}$ & 40 & VS & $1-2$ & $\mathrm{Fe} \mathrm{Bl}$ & 4 & $\begin{array}{c}\text { Other } \\
\text { (psychopathy) }\end{array}$ \\
\hline $\begin{array}{l}\text { Vazire, Naumann, Rentfrow, } \\
\text { \& Gosling (2008) }\end{array}$ & $\begin{array}{l}\mathrm{CV} \\
\mathrm{CU}\end{array}$ & 160 & 7 & $\mathrm{Ph}$ & $<1$ & Fe Ap & 16 & NEA \\
\hline $\begin{array}{l}\text { Zebrowitz, \& Montepare } \\
\text { (1992) }\end{array}$ & $\mathrm{CU}$ & 88 & $\begin{array}{r}118 / \\
48\end{array}$ & $\mathrm{Ph}$ & $\mathrm{P}$ & Ap & 2 & A \\
\hline $\begin{array}{l}\text { Zebrowitz, Hall, Murphy, \& } \\
\text { Rhodes (2002) }\end{array}$ & $\begin{array}{l}\mathrm{CU} \\
\mathrm{CV}\end{array}$ & 502 & 24 & $\mathrm{Ph}$ & $\begin{array}{l}14- \\
32\end{array}$ & Ap & 3 & I \\
\hline $\begin{array}{l}\text { Zebrowitz, Voinescu, \& } \\
\text { Collins (1996) }\end{array}$ & $\mathrm{CU}$ & 103 & 24 & $\mathrm{Ph}$ & $\begin{array}{l}2- \\
72\end{array}$ & Fe Ap & 5 & A \\
\hline
\end{tabular}

Note. Type = Type of research: $\mathrm{CV}=$ Cue validity, $\mathrm{CU}=$ Cue utilization. $N(\mathrm{~T})$ Number of targets.

$N(\mathrm{P})=$ Number of perceivers per target. Jud. Channel = Judgment channel: VS = Video with sound, Vws $=$ Video without sound, $\mathrm{Au}=$ Audio only, $\mathrm{Ph}=$ Photograph only, Inter $=$ Short interaction. $N(\mathrm{R})=$ Number of cue raters per target per cue. Cue types $=$ Domains of cues included in the respective study: $\mathrm{Fe}=$ Facial expression, $\mathrm{Bl}=$ Body language, $\mathrm{Pl}=$ Paralanguage, $\mathrm{Ap}=$ Appearance . No of cues $=$ number of cues identified within the study that exclusively belong to the four cue domains. Traits included in the study, mapped on the Big Five and Intelligence: $\mathrm{N}=$ Neuroticism, E = Extraversion, $\mathrm{O}=$ Openness to experience, $\mathrm{A}=$ Agreeableness, $\mathrm{C}=$ Conscientiousness, $\mathrm{I}=$ Intelligence. 
Table S2.

\section{Allocation of traits and cues to superordinate domains}

\begin{tabular}{|c|c|}
\hline Neuroticism & $\begin{array}{l}\text { Neuroticism; self-consciousness; social anxiety; composure; emotional-unemotional; } \\
\text { emotional stability; self-doubting - self-confident }\end{array}$ \\
\hline Extraversion & $\begin{array}{l}\text { Ambitious-dominant; assertiveness; dominance; engagement/dominance; extraversion; } \\
\text { extroversion; expressive-confident; gregarious-extraverted; leadership competence; } \\
\text { masculinity (BSRI); narcissism (NPI: leadership/authority); other-directedness; power } \\
\text { motive; sociability; submissive - dominant; aloof-introverted; extraverted-introverted; } \\
\text { humorous - serious; inhibition; introversion; lazy-submissive; shyness; sociable - } \\
\text { unsociable }\end{array}$ \\
\hline Openness & $\begin{array}{l}\text { Culture; culture/openness; culture-openness to experience; imaginative; interest/invitation; } \\
\text { openness; openness to experience }\end{array}$ \\
\hline Agreeableness & $\begin{array}{l}\text { Affiliation; affiliative nurturance; agreeableness; femininity (BSRI); good-natured; honesty; } \\
\text { likability; altruism; concern for others; social composite score; teamwork competence; } \\
\text { trustworthiness; unassuming- ingenuous; warm - agreeable; warmth; arrogant-calculating; } \\
\text { cold-quarrelsome; kind-cruel; narcissism (NPI: exploitativeness/entitlement); } \\
\text { phony/arrogant; provocative }\end{array}$ \\
\hline
\end{tabular}

Conscientiousness Conscientiousness; drive; lazy - energetic; planning and organization competence

Intelligence Competence; intelligence; IQ; speaking ability; verbal comprehension test

Cheerful; cheerfulness of facial expression; degree of felt smile; Duchene smiles; (positive) emotional reactions; expression of positivity; extensive smiling; extent of smiling; frequency of smiling; friendliness of facial expression; friendly expression; friendly facial expression;

Cheerful facial expression

Dominant facial expression

Eye contact

Eyebrow movements

General expressiveness

Unconcerned (vs. serious)

Body movement grumpy - friendly expression; happy facial expression; intense smiling; joyfulness; laugh number; laugh rate while not speaking; laughs frequently; no smiling - extensive smiling; non-Duchene smiles; noticeably positive facial expression (smile); open smiles; positive facial expression; smile; smile extent while not speaking; smile extent while speaking; smile number; smile time; smiles; smiling; smiling (time); smiling while listening; smiling while speaking; smiling/facial pleasantness; time per smile; total smiling; wide open smile

facial expression of dominance; self-assured expression; self-assured facial expression; timid - self-assured expression; visual dominance

amount of time spent gazing at others; attend; avoided - engaged in eye contact; engages in constant eye contact; eye contact; eye contact (time); eye gaze at partner; eye-gaze; facial regard; fixation; facial gazing; short glances; frequency of eye contact; frequency of gaze; frequency of gaze while speaking; gaze; gaze mean duration overall; gaze mean duration while speaking; gaze number; gaze rate while not speaking; gaze time; intense look into camera; look while speaking; looking while listening; mean length of eye contact; number of eye contacts; time of looking into the camera; percent looking while listening; percent looking while speaking; total looking; gaze aversion; looking away from camera; downward gaze (time); downward gazes (number)

Brow furrowing; concern furrows; eyebrow flashes (number.); eyebrow flashes and raises; eyebrow lifts; frowns; raised eyebrows

Facial expressions; facial expressiveness; inexpressive - expressive face; unchanging facial expression

Calm facial expression; indifferent expression; serious - unconcerned expression; unconcerned expression; serious face; serious facial expressions

Amount of body movement; body activity; body swaying; fast movements; foot movement; is physically animated; moves around a great deal; leg movement; many body movements; moved around a little - a great deal; number of turning points; overall motion; position 
change; postural shift rate while speaking; rapid body movement; slow - fast movements; speed \& energy of body movement; trunk movements

Closed arms

Forward lean / proximity

Gestures

Head movements

Self-touch

Stride length

Tension / nervousness (vs. relaxed)

Ease of understanding

Expressive/ varying voice

Fluent speaking (vs. nervous)

Interruptions

Loudness

Pitch
Arm wrap; arms folded; closed arms; closed arms while sitting; crossed arms; arms held in an open vs. wrapped position; closed-open arms while sitting

avoided - approached physical contact; body lean; body orientation; forward lean; forward lean (time); makes or approaches physical contact with the partner; makes physical contact with partner; proximity; torso leaning; personal space; trunk recline

Amount of time spent gesturing; broad gestures; degree of arm swinging while walking; energetic gestures; fast gestures; frequent gestures; frequent hand movements; gestural fluency; gestures; gestures while speaking; gesturing; hand gestures (number); hand movement; illustrating hand movements; illustrator duration; illustrator gestures; infrequent - frequent hand movements; number of arm movements; talking with hands; total gesturing; lack of arm swinging

Frequent head movements; head cant; head cants (time); head movements; head moves; head nodding; head nods; head shake; head shakes; infrequent - frequent head movements; nod rate; nodding; nods; upward head tilt; does not tilt head

Body openness; dominant behavior/movements; energetic stance; erect (vs. slouched) posture; leg openness; occupying much space; open posture (time); self-assured body movements; self-assured body posture; self-assuredness of movement; straight posture; upright posture; slouching

Body adaptor duration; body contact (hands in contact with body); facial adaptor duration; primping self-touch (grooming); self-manipulation; self-manipulating (self-touching) hand movements; self-touch; touched own body rarely - frequently; touches oneself frequently; touches self frequently; touching the self; touching the self while listening; touching the self while speaking

Long strides; small steps - long strides; stride length; number of steps to leave; number of steps to chair

Body tension; controlled way of sitting; fidgeting; stiff walking; tense body posture; tense stance; tremors; controlled - relaxed sitting; expansive (vs. constricted); nervous - relaxed movements; relaxed sitting; relaxed way of walking; stiff-relaxed walking

Clear communication; clear style of speech; difficult - easy to understand; ease of understanding; easy to understand; easy to understand speech; enunciates; language that was easy to understand; mumbles - articulates

Articulate voice; dynamic range; energetic and cheerful voice; expressive voice; fundamental vocal frequency (variance); modulation; pitch variety; resonance; resonance of voice; rhythmic speech; vocal intensity (variance); voice animation

Calm voice; calm way of speaking; fluency; pauses; response latencies; fluent speaking; haltingly - fluently speaking; hectic - calm speaking; speaking fluency; speaks fluently; speaks fluently and expresses ideas well; verbal fluency; voice wavers - calm voice; halting speech; haltingly speaking; hectic speaking; hesitation while speaking; nonfluencies; use of fillers during speech; vocal nervousness

Interrupts partner; silent interruptions; simultaneous speech: simple interruptions; simultaneous speech: butting-in-interruptions; simultaneous speech: overlaps; interrupted me frequently - rarely

fundamental vocal frequency (average); high volume of voice; loud voice; loudness; loudness of voice; speaks in a loud voice; loud voiced - soft voiced

Deep - high voice; high pitch; high pitch of voice; high pitched voice; high tone of voice; high voice; pitch; vocal intensity (average) 
Pleasantness of voice

Powerful /confident voice (vs. soft)

Speech rate

Speech vs. nonspeech cheerful tone of voice; friendly tone of voice; pleasant speech style; pleasant voice; pleasantness of voice; unpleasant - pleasant voice; warmth of voice; unpleasant voice confident manner; full voice; harshness of voice; powerful voice; self-assured; sharpness of voice; strength of voice; weak - powerful voice; breathiness of voice; smooth voice; softvoiced; thinness of voice

fast talker; rapid rate; speaks quickly; speech rate; voice rate

Amount of utterances; had not spoken - had spoken a lot; is talkative (as observed in this situation); number of words; sound-silence ratio; speaking time; speech; speech duration; talking; time spent talking; time talking; verbalization; number of silences during conversation

Attractive; attractive face; attractive person; attractiveness; attractiveness of face and body;

Attractiveness facial attractiveness; healthy appearance; physical attractiveness; Ill - well-proportioned body

Babyfaceness vs.

Baby face; babyfaceness; childlike face; facial babyishness; feminine; feminine appearance; maturity hard - soft facial lineaments; perceived age (young); round face; soft-lineaments; childlike mature face; hard facial lineaments; perceived age; round - thin face

Dark clothes

Dark garments; light - dark garments; colorful dress

Dark hair color

Dark hair; dark - light hair; light hair

Distinctiveness

Distinctive appearance; distinctiveness of forehead; distinctiveness of nose; facial averageness

Eyeglasses

Eyeglasses

Formality

Chic dress; formal dress; formality of dress; formally dressed; formal - informal dress; informal dress; outlandishness of dress

Hair length (long) long hair; hair length; short - long hair

Height $\quad$ Body height; stature; short - tall stature; tall stature

Muscularity Less - more muscular physique; muscular; muscular physique; less muscular physique

Neat appearance; neat vs. messy appearance; neatly dressed; neatness; neatness of dress; Neatness $\quad$ organized appearance; refined appearance; trimness; unrefined - refined appearance; wellgroomed face; unrefined appearance

Dressed-up person; expensive clothes; fashionable and refined appearance; fashionable body \& dress; fashionable clothes; fashionable dress; fashionable face \& hairstyle; flashy

Stylishness accessories; made-up face; not made up - made up face; plain - showy dress; showy dress; stylish clothes; stylish appearance; stylish dress; stylish hair; stylishness; trendy hairstyle; unfashionable - fashionable dress; unstylish - stylish hair; wears much jewelry; plain clothes; unfashionable dress

Volume of Mouth / Thin - full lips; volume of mouth; thin lips full lips

Weight Body size; stout physique; weight; low body weight; stout - slim physique

Note. BSRI = Bem Sex-Role-Inventory. NPI = Narcissistic Personality Inventory. The allocation

process was done by the authors of this study and based on theoretical and practical

considerations. Italicized cues were reverse scored. 
Table S3.

Vector correlations of cue utilization and cue validity within and across traits

\begin{tabular}{|c|c|c|c|c|c|c|c|c|c|c|c|}
\hline & 2 & 3 & 4 & 5 & 6 & 7 & 8 & 9 & 10 & 11 & 12 \\
\hline 1. CU Emotional stability & .47 & .72 & .63 & .63 & .05 & .50 & -.05 & .34 & .28 & .63 & .40 \\
\hline 2. CV Emotional stability & & .56 & .56 & .30 & -.22 & .33 & -.23 & .17 & .41 & .45 & -.15 \\
\hline 3. CU Extraversion & & & .81 & .62 & -.07 & .60 & .06 & .14 & .33 & .67 & .26 \\
\hline 4. CV Extraversion & & & & .42 & -.12 & .40 & .01 & .28 & .54 & .62 & .14 \\
\hline 5. CU Openness & & & & & .37 & .72 & .38 & .39 & .28 & .55 & .24 \\
\hline 6. CV Openness & & & & & & .16 & .24 & -.22 & -.27 & -.13 & .25 \\
\hline 7. CU Agreeableness & & & & & & & .42 & .41 & .27 & .36 & .12 \\
\hline 8. CV Agreeableness & & & & & & & & .39 & .16 & .11 & -.11 \\
\hline 9. CU Conscientiousness & & & & & & & & & .69 & .34 & -.07 \\
\hline 10. CV Conscientiousness & & & & & & & & & & .43 & -.21 \\
\hline 11. CU Intelligence & & & & & & & & & & & .41 \\
\hline 12. CV Intelligence & & & & & & & & & & & \\
\hline
\end{tabular}

Note. $\mathrm{CU}=$ Cue utilization. $\mathrm{CV}=$ Cue validity. Number of cues range from 31 to 39. 


\section{References}

Albright, L., Kenny, D. A., \& Malloy, T. E. (1988). Consensus in personality judgments at zero acquaintance. Journal of Personality and Social Psychology, 55(3), 387-395.

https://doi.org/10.1037/0022-3514.55.3.387

Albright, L., Malloy, T. E., Dong, Q., Kenny, D. A., Fang, X., Winquist, L., Yu, D. (1997). Crosscultural consensus in personality judgments. Journal of Personality and Social Psychology, 72(3), 558-569. https://doi.org/10.1037//0022-3514.72.3.558

Ambady, N., Bernieri, F. J., \& Richeson, J. A. (2000). Toward a histology of social behaviorjudgmental accuracy from thin slices of the behavioral stream. Advances in Experimental Social Psychology, 32, 201-271.

Ambady, N., \& Skowronski, J. J. (Eds.). (2008). First impressions. New York: Guilford Press.

Aronovitch, C. D. (1976). The voice of personality: Stereotyped judgments and their relation to voice quality and sex of speaker. Journal of Social Psychology, 99, 207-220.

Asendorpf, J. B., Banse, R., \& Mücke, D. (2002). Double dissociation between implicit and explicit personality self-concept: The case of shy behavior. Journal of Personality and Social Psychology, 83(2), 380-393. https://doi.org/10.1037//0022-3514.83.2.380

Back, M. D., \& Nestler, S. (2016). Accuracy of judging personality. In J. A. Hall, M. S. Mast, \& T. V. West (Eds.), The social psychology of perceiving others accurately (98-124). Cambridge: Cambridge University Press.

Back, M. D., Penke, L., Schmukle, S. C., Sachse, K., Borkenau, P., \& Asendorpf, J. B. (2011). Why mate choices are not as reciprocal as we assume: The role of personality, flirting and physical attractiveness. European Journal of Personality, 25(2), 120-132. https://doi.org/10.1002/per.806

Back, M. D., Schmukle, S. C., \& Egloff, B. (2008). How extraverted is honey.bunny77@ hotmail.de? Inferring personality from e-mail addresses. Journal of Research in Personality, 42(4), 11161122. https://doi.org/10.1016/j.jrp.2008.02.001 
Back, M. D., Schmukle, S. C., \& Egloff, B. (2010). Why are narcissists so charming at first sight? Decoding the narcissism-popularity link at zero acquaintance. Journal of Personality and Social Psychology, 98(1), 132-145. https://doi.org/10.1037/a0016338

Back, M. D., Schmukle, S. C., \& Egloff, B. (2011). A closer look at first sight: Social relations lens model analysis of personality and interpersonal attraction at zero acquaintance. European Journal of Personality, 25(3), 225-238. https://doi.org/10.1002/per.790

Back, M. D., Stopfer, J. M., Vazire, S., Gaddis, S., Schmukle, S. C., Egloff, B., \& Gosling, S. D. (2010). Facebook profiles reflect actual personality, not self-idealization. Psychological Science, 21(3), 372-374. https://doi.org/10.1177/0956797609360756

Bakemann, R. (2000). Behavioral observation and coding. In H. T. Reis \& C. M. Judd (Eds.), Handbook of research methods in social and personality psychology (pp. 138-159). New York: Cambridge University Press.

Bänziger, T., Hosoya, G., \& Scherer, K. R. (2015). Path Models of Vocal Emotion Communication. PloS One, 10(9), e0136675. https://doi.org/10.1371/journal.pone.0136675

Berry, D. S. (1990). Taking people at face value: Evidence for the kernel of truth hypothesis. Social Cognition, 8(4), 343-361.

Berry, D. S., \& Hansen, J. S. (2000). Personality, nonverbal behavior, and interaction quality in female dyads. Personality and Social Psychology Bulletin, 26(3), 278-292. https://doi.org/10.1177/0146167200265002

Berry, D. S., \& Landry, J. C. (1997). Facial maturity and daily social interaction. Journal of Personality and Social Psychology, 72(3), 570-580. https://doi.org/10.1037/00223514.72.3.570

Biel, J.-I., Aran, O., \& Gatica-Perez, D. (2011). You are known by how you vlog: Personality impressions and nonverbal behavior in YouTube. Proceedings of the Fifth International AAAI Conference on Weblogs and Social Media, 446-449.

Blanck, P. D., Rosenthal, R., Snodgrass, S. E., DePaulo, B. M., \& Zuckerman, M. (1981). Sex differences in eavesdropping on nonverbal cues: Developmental changes. Journal of 
Personality and Social Psychology, 41(2), 391-396. https://doi.org/10.1037//0022-

3514.41.2.391

Borkenau, P., Brecke, S., Möttig, C., \& Paelecke, M. (2009). Extraversion is accurately perceived after a 50-ms exposure to a face. Journal of Research in Personality, 43(4), 703-706. https://doi.org/10.1016/j.jrp.2009.03.007

Borkenau, P., \& Liebler, A. (1992a). The cross-modal consistency of personality: Inferring strangers' traits from visual or acoustic information. Journal of Research in Personality, 26(2), 183-204. https://doi.org/10.1016/0092-6566(92)90053-7

Borkenau, P., \& Liebler, A. (1992b). Trait inferences: Sources of validity at zero acquaintance. Journal of Personality and Social Psychology, 62(4), 645-657. https://doi.org/10.1037/00223514.62.4.645

Borkenau, P., \& Liebler, A. (1993). Convergence of stranger ratings of personality and intelligence with self-ratings, partner ratings, and measuerd intelligence. Journal of Personality and Social Psychology, 65(3), 546-553.

Borkenau, P., \& Liebler, A. (1995). Observable attributes as manifestations and cues of personality and intelligence. Journal of Personality, 63(1), 1-25. https://doi.org/10.1111/j.14676494.1995.tb00799.x

Borkenau, P., Mauer, N., Riemann, R., Spinath, F. M., \& Angleitner, A. (2004). Thin slices of behavior as cues of personality and intelligence. Journal of Personality and Social Psychology, 86(4), 599-614. https://doi.org/10.1037/0022-3514.86.4.599

Borkenau, P., Mosch, A., Tandler, N., \& Wolf, A. (2016). Accuracy of judgments of personality based on textual information on major life domains. Journal of Personality, 84(2), 214-224. https://doi.org/10.1111/jopy.12153

Brauner, E., Boos, M., \& Kolbe, M. (Eds.). (2018). Cambridge Handbook of Group Interaction Analysis. Cambridge: Cambridge University Press.

Brown, W. M., Palameta, B., \& Moore, C. (2003). Are there nonverbal cues to commitment? An exploratory study using the zero-acquaintance video presentation paradigm. Evolutionary Psychology, 1(1), 42-69. https://doi.org/10.1177/147470490300100104 
Brunswik, E. (1952). The conceptual framework of psychology. International encyclopedia of unified science: v. 1, no. 10. [Chicago]: Univ. of Chicago Press.

Brunswik, E. (1956). Perception and the representative design of psychological experiments (2nd ed.). Berkeley: University of California Press.

Burgoon, J. K., Birk, T., \& Pfau, M. (1990). Nonverbal behaviors, persuasion, and credibility. Human Communication Research, 17(1), 140-169. https://doi.org/10.1111/j.1468-2958.1990.tb00229.x

Burgoon, J. K., Guerrero, L. K., \& Floyd, K. (2010). Nonverbal communication. London: Routledge, Taylor \& Francis Group.

Burnett, J. R., \& Motowidlo, S. J. (1998). Relations between different sources of information in the structured selection interview. Personnel Psychology, 51(4), 963-983. https://doi.org/10.1111/j.1744-6570.1998.tb00747.x

Campbell, A., \& Rushton, J. P. (1978). Bodily communication and personality. British Journal of Social and Clinical Psychology, 17(1), 31-36. https://doi.org/10.1111/j.20448260.1978.tb00893.x

Carney, D. R., Hall, J. A., \& LeBeau, L. S. (2005). Beliefs about the nonverbal expression of social power. Journal of Nonverbal Behavior, 29(2), 105-123. https://doi.org/10.1007/s10919-005$2743-\mathrm{z}$

Cohen, J. (1992). A power primer. Psychological Bulletin, 112(1), 155-159. https://doi.org/10.1037//0033-2909.112.1.155

Colvin, C. R. (1993). "Judgable" people: Personality, behavior, and competing explanations. Journal of Personality and Social Psychology, 64(5), 861-873. https://doi.org/10.1037//00223514.64.5.861

Connelly, B. S., \& Ones, D. S. (2010). An other perspective on personality: meta-analytic integration of observers' accuracy and predictive validity. Psychological Bulletin, 136(6), 1092-1122. https://doi.org/10.1037/a0021212

Connolly, J. J., Kavanagh, E. J., \& Viswesvaran, C. (2007). The convergent validity between self and observer ratings of personality: A meta-analytic review. International Journal of Selection and Assessment, 15(1), 110-117. https://doi.org/10.1111/j.1468-2389.2007.00371.x 
Creed, A. T., \& Funder, D. C. (1998). Social anxiety: from the inside and outside. Personality and Individual Differences, 25(1), 19-33. https://doi.org/10.1016/S0191-8869(98)00037-3

Darwin, C. (1897). The expression of the emotions in man and animals. New York: D. Appleton and Company.

Davis, M. H., \& Kraus, L. A. (1997). Personality and empathic accuracy. In W. Ickes (Ed.), Empathic accuracy (pp. 144-168). New York, Guilford.

DeGroot, T., \& Gooty, J. (2009). Can nonverbal cues be used to make meaningful personality attributions in employment interviews? Journal of Business and Psychology, 24(2), 179-192. https://doi.org/10.1007/s10869-009-9098-0

DePaulo, B. M. (1992). Nonverbal behavior and self-presentation. Psychological Bulletin, 111(2), 203-243. https://doi.org/10.1037//0033-2909.111.2.203

Eaton, L. G., \& Funder, D. C. (2003). The creation and consequences of the social world: an interactional analysis of extraversion. European Journal of Personality, 17(5), 375-395. https://doi.org/10.1002/per.477

Ekman, P. (1994). Strong evidence for universals in facial expressions: A reply to Russell's mistaken critique. Psychological Bulletin, 115(2), 268-287.

Ekman, P. (2016). What scientists who study emotion agree about. Perspectives on Psychological Science, 11(1), 31-34. https://doi.org/10.1177/1745691615596992

Ekman, P., \& Friesen, W. V.,. (1969). Nonverbal leakage and clues to deception. Psychiatry, 32(1), $87-106$.

Ekman, P., \& Friesen, W. V. (1971). Constants across cultures in the face and emotion. Journal of Personality and Social Psychology, 17(2), 124-129. https://doi.org/10.1037/h0030377

Ekman, P., \& Rosenberg, E. L. (Eds.). (1997). Series in affective science. What the face reveals: Basic and applied studies of spontaneous expression using the facial action coding system (FACS). New York, Oxford: Oxford University Press.

Ekman, P., Sorenson, E. R., \& Friesen, W. V. (1969). Pan-cultural elements in facial displays of emotion. Science, 164(3875), 86-88. https://doi.org/10.1126/science.164.3875.86 
Elfenbein, H. A., \& Eisenkraft, N. (2010). The relationship between displaying and perceiving nonverbal cues of affect: a meta-analysis to solve an old mystery. Journal of Personality and Social Psychology, 98(2), 301-318. https://doi.org/10.1037/a0017766

Ferguson, N. (1977). Simultaneous speech, interruptions and dominance. British Journal of Social and Clinical Psychology, 16(4), 295-302. https://doi.org/10.1111/j.2044-8260.1977.tb00235.x

Funder, D. C. (1999). Personality judgment: A realistic approach to person perception. San Diego, CA: Academic Press.

Funder, D. C. (2012). Accurate personality judgment. Current Directions in Psychological Science, 21(3), 177-182. https://doi.org/10.1177/0963721412445309

Funder, D. C., \& Sneed, C. D. (1993). Behavioral manifestations of personality: An ecological approach to judgmental accuracy. Journal of Personality and Social Psychology, 64(3), 479490. https://doi.org/10.1037//0022-3514.64.3.479

Gifford, R. (1991). Mapping nonverbal behavior on the interpersonal circle. Journal of Personality and Social Psychology, 61(2), 279-288. https://doi.org/10.1037/0022-3514.61.2.279

Gifford, R. (1994). A lens-mapping framework for understanding the encoding and decoding of interpersonal dispositions in nonverbal behavior. Journal of Personality and Social Psychology, 66(2), 398-412. https://doi.org/10.1037//0022-3514.66.2.398

Gifford, R., Ng, C. F., \& Wilkinson, M. (1985). Nonverbal cues in the employment interview: Links between applicant qualities and interviewer judgments. Journal of Applied Psychology, 70(4), 729-736. https://doi.org/10.1037//0021-9010.70.4.729

Gifford, R., \& O'Connor, B. (1987). The interpersonal circumplex as a behavior map. Journal of Personality and Social Psychology, 52(5), 1019-1026. https://doi.org/10.1037/00223514.52.5.1019

Gillath, O., Bahns, A. J., Ge, F., \& Crandall, C. S. (2012). Shoes as a source of first impressions. Journal of Research in Personality, 46(4), 423-430. https://doi.org/10.1016/j.jrp.2012.04.003

Gosling, S. D., Ko, S. J., Mannarelli, T., \& Morris, M. E. (2002). A room with a cue: Personality judgments based on offices and bedrooms. Journal of Personality and Social Psychology, 82(3), 379-398. https://doi.org/10.1037/0022-3514.82.3.379 
Grünberg, M., Mattern, J., Geukes, K., Küfner, A. C. P., \& Back, M. D. (2018). Assessing group interactions in personality psychology: The Münster Behavior Coding-System (M-BeCoSy). In E. Brauner, M. Boos, \& M. Kolbe (Eds.), Cambridge Handbook of Group Interaction Analysis (pp. 602-611). Cambridge: Cambridge University Press.

Hall, J. A., \& Andrzejewski, S. A. (2008). Who draws accurate first impressions?: Personal correlates of sensitivitiy to nonverbal cues. In N. Ambady \& J. J. Skowronski (Eds.), First impressions (pp. 87-105). New York: Guilford Press.

Hall, J. A., Andrzejewski, S. A., Murphy, N. A., Mast, M. S., \& Feinstein, B. A. (2008). Accuracy of judging others' traits and states: Comparing mean levels across tests. Journal of Research in Personality, 42(6), 1476-1489. https://doi.org/10.1016/j.jrp.2008.06.013

Hall, J. A., Andrzejewski, S. A., \& Yopchick, J. E. (2009). Psychosocial correlates of interpersonal sensitivity: A meta-analysis. Journal of Nonverbal Behavior, 33(3), 149-180. https://doi.org/10.1007/s10919-009-0070-5

Hall, J. A., \& Bernieri, F. J. (2001). Interpersonal sensitivity: Theory and measurement. Mahwah, NJ: Erlbaum.

Hall, J. A., Coats, E. J., \& LeBeau, L. S. (2005). Nonverbal behavior and the vertical dimension of social relations: a meta-analysis. Psychological Bulletin, 131(6), 898-924. https://doi.org/10.1037/0033-2909.131.6.898

Hall, J. A., Gunnery, S. D., Letzring, T. D., Carney, D. R., \& Colvin, C. R. (2017). Accuracy of judging affect and accuracy of judging personality: How and when are they related? Journal of Personality, 85(5), 583-592. https://doi.org/10.1111/jopy.12262

Hall, J. A., Horgan, T. G., \& Murphy, N. A. (2018). Nonverbal communication. Annual Review of Psychology. Advance online publication. https://doi.org/10.1146/annurev-psych-010418103145

Hall, J. A., Schmidt Mast, M., \& West, T. V. (2016). Accurate interpersonal perception: Many traditions, one topic. In J. A. Hall, M. S. Mast, \& T. V. West (Eds.), The social psychology of perceiving others accurately (pp. 3-22). Cambridge: Cambridge University Press. 
Harrigan, J. A., Rosenthal, R., \& Scherer, K. R. (Eds.). (2005). Series in affective science. The new handbook of methods in nonverbal behavior research. Oxford: Oxford University Press.

Harris, M. J., \& Garris, C. P. (2008). You never get a second chance to make a first impression: Behavioral consequences of first impressions. In N. Ambady \& J. J. Skowronski (Eds.), First impressions (pp. 147-168). New York: Guilford Press.

Hartung, F.-M., \& Renner, B. (2011). Social curiosity and interpersonal perception: A judge x trait interaction. Personality and Social Psychology Bulletin, 37(6), 796-814. https://doi.org/10.1177/0146167211400618

Hassin, R., \& Trope, Y. (2000). Facing faces: Studies on the cognitive aspects of physiognomy. Journal of Personality and Social Psychology, 78(5), 837-852. https://doi.org/10.1037/00223514.78.5.837

Henley, N. (1977). Body politics: Power, sex, and nonverbal communication. New York: Simon \& Schuster.

Heyman, R. E., Lorber, M. F., Eddy, J. M., \& West, T. V. (2014). Behavioral observation and coding. In H. T. Reis \& C. M. Judd (Eds.), Handbook of research methods in social and personality psychology (2nd ed., pp. 345-372). New York: Cambridge University Press. https://doi.org/10.1017/CBO9780511996481.018

Hildebrandt, A., Olderbak, S., \& Wilhelm, O. (2015). Facial emotion expression, individual differences in. In International Encyclopedia of the Social \& Behavioral Sciences (pp. 667675). Elsevier. https://doi.org/10.1016/B978-0-08-097086-8.25008-3

Hirschmüller, S., Egloff, B., Nestler, S., \& Back, M. D. (2013). The dual lens model: A comprehensive framework for understanding self-other agreement of personality judgments at zero acquaintance. Journal of Personality and Social Psychology, 104(2), 335-353. https://doi.org/10.1037/a0030383

Hirschmüller, S., Egloff, B., Schmukle, S. C., Nestler, S., \& Back, M. D. (2015). Accurate judgments of neuroticism at zero acquaintance: a question of relevance. Journal of Personality, 83(2), 221228. https://doi.org/10.1111/jopy.12097 
Hirschmüller, S., Schmukle, S. C., Krause, S., Back, M. D., \& Egloff, B. (2018). Accuracy of selfesteem judgments at zero acquaintance. Journal of Personality, 86(2), 308-319. https://doi.org/10.1111/jopy.12316

Human, L. J., \& Biesanz, J. C. (2011). Target adjustment and self-other agreement: Utilizing trait observability to disentangle judgeability and self-knowledge. Journal of Personality and Social Psychology, 101(1), 202-216. https://doi.org/10.1037/a0023782

Human, L. J., Biesanz, J. C., Finseth, S. M., Pierce, B., \& Le, M. (2014). To thine own self be true: Psychological adjustment promotes judgeability via personality-behavior congruence. Journal of Personality and Social Psychology, 106(2), 286-303. https://doi.org/10.1037/a0034860

Ickes, W., \& Barnes, R. D. (1977). The role of sex and self-monitoring in unstructured dyadic interactions. Journal of Personality and Social Psychology, 35(5), 315-330. https://doi.org/10.1037/0022-3514.35.5.315

Izard, C. E. (1971). The face of emotion. The century psychology series. New York.

Izard, C. E. (1994). Innate and universal facial expressions: Evidence from developmental and crosscultural research. Psychological Bulletin, 115(2), 288-299. https://doi.org/10.1037//00332909.115.2.288

Jackson, L. A., Hunter, J. E., \& Hodge, C. N. (1995). Physical attractiveness and intellectual competence: A meta-analytic review. Social Psychology Quarterly, 58(2), 108-122.

Jussim, L., Cain, T., Crawford, J., Harber, K., \& Cohen, F. (2009). The unbearable accuracy of stereotypes. In T. Nelson (Ed.), Handbook of prejudice, stereotyping, and discrimination (pp. 199-227). Hillsdale, NJ: Erlbaum.

Kanazawa, S. (2011). Intelligence and physical attractiveness 25. Intelligence, 39(1), 7-14. https://doi.org/10.1016/j.intell.2010.11.003

Kaurin, A., Heil, L., Wessa, M., Egloff, B., \& Hirschmüller, S. (2018). Selfies reflect actual personality - Just like photos or short videos in standardized lab conditions. Journal of Research in Personality, 76, 154-164. https://doi.org/10.1016/j.jrp.2018.08.007

Kendon, A., \& Cook, M. (1969). The consistency of gaze patterns in social interaction. British Journal of Psychology, 60(4), 481-494. 
Kenny, D. A., Albright, L., Malloy, T. E., \& Kashy, D. A. (1994). Consensus in interpersonal perception: Acquaintance and the big five. Psychological Bulletin, 116(2), 245-258. https://doi.org/10.1037/0033-2909.116.2.245

Kenny, D. A., Horner, C., Kashy, D. A., \& Chu, L.-c. (1992). Consensus at zero acquaintance: Replication, behavioral cues, and stability. Journal of Personality and Social Psychology, 62(1), 88-97. https://doi.org/10.1037//0022-3514.62.1.88

Knutson, B. (1996). Facial expressions of emotion influence interpersonal trait inferences. Journal of Nonverbal Behavior, 20(3), 165-182. https://doi.org/10.1007/BF02281954

Koppensteiner, M., \& Grammer, K. (2010). Motion patterns in political speech and their influence on personality ratings. Journal of Research in Personality, 44(3), 374-379. https://doi.org/10.1016/j.jrp.2010.04.002

Koppensteiner, M., Stephan, P., \& Jäschke, J. P. M. (2016). Moving speeches: Dominance, trustworthiness and competence in body motion. Personality and Individual Differences, 94, 101-106. https://doi.org/10.1016/j.paid.2016.01.013

Kramer, E. (1963). Judgment of personal characteristics and emotions from nonverbal properties of speech. Psychological Bulletin, 60(4), 408-420. https://doi.org/10.1037/h0044890

Küfner, A. C.P., Back, M. D., Nestler, S., \& Egloff, B. (2010). Tell me a story and I will tell you who you are! Lens model analyses of personality and creative writing. Journal of Research in Personality, 44(4), 427-435. https://doi.org/10.1016/j.jrp.2010.05.003

Langlois, J. H., Kalakanis, L., Rubenstein, A. J., Larson, A., Hallam, M., \& Smoot, M. (2000). Maxims or myths of beauty? A meta-analytic and theoretical review. Psychological Bulletin, 126(3), 390-423. https://doi.org/10.1037//0033-2909.126.3.390

Letzring, T. D. (2008). The good judge of personality: Characteristics, behaviors, and observer accuracy. Journal of Research in Personality, 42(4), 914-932. https://doi.org/10.1016/j.jrp.2007.12.003

Letzring, T. D., Wells, S. M., \& Funder, D. C. (2006). Information quantity and quality affect the realistic accuracy of personality judgment. Journal of Personality and Social Psychology, 91(1), 111-123. https://doi.org/10.1037/0022-3514.91.1.111 
Levesque, M. J., \& Kenny, D. A. (1993). Accuracy of behavioral predictions at zero acquaintance: A social relations analysis. Journal of Personality and Social Psychology, 65(6), 1178-1187. https://doi.org/10.1037/0022-3514.65.6.1178

Lippa, R. (1998). The nonverbal display and judgment of extraversion, masculinity, femininity, and gender diagnosticity: A lens model analysis. Journal of Research in Personality, 32(1), 80-107. https://doi.org/10.1006/jrpe.1997.2189

Lyons, K. D., Tickle-Degnen, L., Henry, A., \& Cohn, E. (2004). Impressions of personality in parkinson's disease: Can rehabilitation practitioners see beyond the symptoms? Rehabilitation Psychology, 49(4), 328-333. https://doi.org/10.1037/0090-5550.49.4.328

Mallory, E. B., \& Miller, V. R. (1958). A possible basis for the association of voice characteristics and personality traits. Speech Monographs, 25(4), 255-260. https://doi.org/10.1080/03637755809375240

Manusov, V. L. (Ed.). (2004). The sourcebook of nonverbal measures: Going beyond words (Digital print). New York, London: Routledge.

Meier, B. P., Robinson, M. D., Carter, M. S., \& Hinsz, V. B. (2010). Are sociable people more beautiful? A zero-acquaintance analysis of agreeableness, extraversion, and attractiveness. Journal of Research in Personality, 44(2), 293-296. https://doi.org/10.1016/j.jrp.2010.02.002

Mitchem, D. G., Zietsch, B. P., Wright, M. J., Martin, N. G., Hewitt, J. K., \& Keller, M. C. (2015). No relationship between intelligence and facial attractiveness in a large, genetically informative sample. Evolution and Human Behavior, 36(3), 240-247.

https://doi.org/10.1016/j.evolhumbehav.2014.11.009

Montepare, J. M., \& Dobish, H. (2003). The contribution of emotion perceptions and their overgeneralizations to trait impressions. Journal of Nonverbal Behavior, 27(4), 237-254.

Murphy, N. A. (2007). Appearing smart: the impression management of intelligence, person perception accuracy, and behavior in social interaction. Personality and Social Psychology Bulletin, 33(3), 325-339. https://doi.org/10.1177/0146167206294871 
Murphy, N. A., Hall, J. A., \& Colvin, C. R. (2003). Accurate intelligence assessments in social interactions: Mediators and gender effects. Journal of Personality, 71(3), 465-493. https://doi.org/10.1111/1467-6494.7103008

Naumann, L. P., Vazire, S., Rentfrow, P. J., \& Gosling, S. D. (2009). Personality judgments based on physical appearance. Personality and Social Psychology Bulletin, 35(12), 1661-1671. https://doi.org/10.1177/0146167209346309

Nestler, S., \& Back, M. D. (2017). Using cross-classified structural equation models to examine the accuracy of personality judgments. Psychometrika, 82(2), 475-497. https://doi.org/10.1007/s11336-015-9485-6

Nestler, S., Egloff, B., Küfner, A. C. P., \& Back, M. D. (2012). An integrative lens model approach to bias and accuracy in human inferences: Hindsight effects and knowledge updating in personality judgments. Journal of Personality and Social Psychology, 103(4), 689-717. https://doi.org/10.1037/a0029461

Nowicki, S., \& Duke, M. P. (1994). Individual differences in the nonverbal communication of affect: The diagnostic analysis of nonverbal accuracy scale. Journal of Nonverbal Behavior, 18(1), 935. https://doi.org/10.1007/BF02169077

Pedersen, D. M. (1973). Correlates of behavioral personal space. Psychological Reports, 32, 828-830. Penke, L., \& Asendorpf, J. B. (2008). Beyond global sociosexual orientations: A more differentiated look at sociosexuality and its effects on courtship and romantic relationships. Journal of Personality and Social Psychology, 95(5), 1113-1135. https://doi.org/10.1037/00223514.95.5.1113

Petrican, R., Todorov, A., \& Grady, C. (2014). Personality at face value: Facial appearance predicts self and other personality judgments among strangers and spouses. Journal of Nonverbal Behavior, 38(2), 259-277. https://doi.org/10.1007/s10919-014-0175-3

Rentfrow, P. J., \& Gosling, S. D. (2006). Message in a ballad: The role of music preferences in interpersonal perception. Psychological Science, 17(3), 236-242. https://doi.org/10.1111/j.14679280.2006.01691.x 
Reynolds, D.'A. J., \& Gifford, R. (2001). The sounds and sights of intelligence: A lens model channel analysis. Personality and Social Psychology Bulletin, 27(2), 187-200.

https://doi.org/10.1177/0146167201272005

Riggio, R. E., \& Friedman, H. S. (1986). Impression formation: The role of expressive behavior. Journal of Personality and Social Psychology, 50(2), 421-427. https://doi.org/10.1037//00223514.50.2.421

Riggio, R. E., Lippa, R., \& Salinas, C. (1990). The display of personality in expressive movement. Journal of Research in Personality, 24(1), 16-31. https://doi.org/10.1016/0092-6566(90)90003$\mathrm{O}$

Scherer, K. R. (1978). Personality inference from voice quality: The loud voice of extroversion. European Journal of Social Psychology, 8(4), 467-487. https://doi.org/10.1002/ejsp.2420080405

Scherer, K. R., Scherer, U., Hall, J. A., \& Rosenthal, R. (1977). Differential attribution of personality based on multi-channel presentation of verbal and nonverbal cues. Psychological Research, 39(3), 221-247. https://doi.org/10.1007/BF00309288

Schmid Mast, M., \& Hall, J. A. (2004). Who is the boss and who is not? Accuracy of judging status. Journal of Nonverbal Behavior, 28(3), 145-165. https://doi.org/10.1023/B:JONB.0000039647.94190.21

Schultheis, O. C., \& Brunstein, J. C. (2002). Inhibited power motivation and persuasive communication: A lens model analysis. Journal of Personality, 70(4), 553-582. https://doi.org/10.1111/1467-6494.05014

Schwarzer, G. (2019). meta: General Package for Meta-Analysis. Retrieved from https://cran.rproject.org/web/packages/meta/index.html

Secord, P. (1958). Facial features and inference processes in interpersonal perception. In R. Tagiuri \& L. Petrullo (Eds.), Person perception and interpersonal behavior (pp. 300-315). Stanford, CA: Stanford University Press.

Shrout, P. E., \& Fiske, D. W. (1981). Nonverbal behaviors and social evaluation. Journal of Personality, 49(2), 115-128. 
Simpson, J. A., Gangestad, S. W., \& Biek, M. (1993). Personality and nonverbal social behavior: An ethological perspective of relationship initiation. Journal of Experimental Social Psychology, $29,434-461$.

Smith, E. R., \& Zárate, M. A. (1992). Exemplar-based model of social judgment. Psychological Review, 99(1), 3-21. https://doi.org/10.1037/0033-295X.99.1.3

Stopfer, J. M., Egloff, B., Nestler, S., \& Back, M. D. (2014). Personality expression and impression formation in online social networks: An integrative approach to Understanding the processes of accuracy, impression management and meta-accuracy. European Journal of Personality, 28(1), 73-94. https://doi.org/10.1002/per.1935

Taft, R. (1955). The ability to judge people. Psychological Bulletin, 52(1), 1-23. https://doi.org/10.1037/h0044999

ten Brinke, L., Porter, S., Korva, N., Fowler, K., Lilienfeld, S. O., \& Patrick, C. J. (2017). An examination of the communication styles associated with psychopathy and their influence on observer impressions. Journal of Nonverbal Behavior, 41(3), 269-287. https://doi.org/10.1007/s10919-017-0252-5

Vazire, S., Naumann, L. P., Rentfrow, P. J., \& Gosling, S. D. (2008). Portrait of a narcissist: Manifestations of narcissism in physical appearance. Journal of Research in Personality, 42(6), 1439-1447. https://doi.org/10.1016/j.jrp.2008.06.007

Willis, J., \& Todorov, A. (2006). First impressions making up your mind after a 100-ms exposure to a face. Psychological Science, 17(7), 592-298.

Youyou, W., Kosinski, M., \& Stillwell, D. (2015). Computer-based personality judgments are more accurate than those made by humans. Proceedings of the National Academy of Sciences of the United States of America, 112(4), 1036-1040. https://doi.org/10.1073/pnas.1418680112

Zebrowitz, L. A. (1997). Reading faces : Window to the soul? Colorado: Weskview Press.

Zebrowitz, L. A. (2017). First impressions from faces. Current Directions in Psychological Science, 26(3), 237-242. https://doi.org/10.1177/0963721416683996 
Zebrowitz, L. A., \& Collins, M. A. (1997). Accurate social perception at zero acquaintance: The affordances of a gibsonian approach. Personality and Social Psychology Review, 1(3), 204-223. https://doi.org/10.1207/s15327957pspr0103_2

Zebrowitz, L. A., Hall, J. A., Murphy, N. A., \& Rhodes, G. (2002). Looking smart and looking good: Facial cues to intelligence and their origins. Personality and Social Psychology Bulletin, 28(2), 238-249. https://doi.org/10.1177/0146167202282009

Zebrowitz, L. A., \& Montepare, J. M. (1992). Impressions of babyfaced individuals across the life span. Developmental Psychology, 28(6), 1143-1152. https://doi.org/10.1037//00121649.28.6.1143

Zebrowitz, L. A., \& Montepare, J. M. (2008). First impressions from facial appearance cues. In N. Ambady \& J. J. Skowronski (Eds.), First impressions (pp. 171-204). New York: Guilford Press.

Zebrowitz, L. A., Voinescu, L., \& Collins, M. A. (1996). "Wide-eyed" and "crooked-faced": Determinants of perceived and real honesty across the life span. Personality and Social Psychology Bulletin, 22(12), 1258-1269. 